\title{
OPTIMAL ERROR ESTIMATES OF THE LOCAL DISCONTINUOUS GALERKIN METHOD FOR WILLMORE FLOW OF GRAPHS ON CARTESIAN MESHES
}

\author{
LIANGYUE JI AND YAN XU
}

\begin{abstract}
In this paper, we analyze a local discontinuous Galerkin method for the willmore flow of graphs. We derive the optimal error estimates for this nonlinear equation in one-dimension and in multi-dimensions for Cartesian meshes using completely discontinuous piecewise polynomial space with degree $k \geq 1$.
\end{abstract}

Key Words. local discontinuous Galerkin method, Willmore flow of graphs, stability, error estimates

\section{Introduction}

In this paper, we consider the error estimates of the local discontinuous Galerkin (LDG) method [23] for the Willmore flow of graphs

$$
u_{t}+Q \nabla \cdot\left(\frac{1}{Q}\left(\boldsymbol{I}-\frac{\nabla u \otimes \nabla u}{Q^{2}}\right) \nabla(Q H)\right)-\frac{1}{2} Q \nabla \cdot\left(\frac{H^{2}}{Q} \nabla u\right)=0,
$$

where $Q$ is the area element

$$
Q=\sqrt{1+|\nabla u|^{2}}
$$

and $H$ is the mean curvature of the domain boundary $\Gamma$

$$
H=\nabla \cdot\left(\frac{\nabla u}{Q}\right) \text {. }
$$

In [23], we developed a LDG method for the for the Willmore flow of graphs and gave a rigorous proof for its energy stability. In this method the basis functions used are discontinuous in space. The LDG discretization also results in a high order accurate, extremely local, element based discretization. In particular, the LDG method is well suited for $h p$-adaptation, which consists of local mesh refinement and/or the adjustment of the polynomial order in individual elements. In this paper, we will present the optimal error analysis for the LDG method of the Willmore flow of graphs on Cartesian meshes. The analysis is made for the fully nonlinear case and the results are valid for all space dimension $d \leq 3$ and polynomial degree $k \geq 1$. We generalize the analysis to fully nonlinear case comparing with analysis for linear fourth order equation in [13]. We also obtain the optimal accuracy results comparing with the results for continuous linear finite element method in [12].

The DG method is a class of finite element methods, using discontinuous, piecewise polynomials as the solution and the test space. It was first designed as a

Received by the editors September 25, 2009 and, in revised form, August 8, 2010.

2000 Mathematics Subject Classification. 65M60, 35K55.

Research of the second author is supported by NSFC grant 10971211, FANEDD, FANEDD of CAS and the Fundamental Research Funds for the Central Universities. Additional support is provided by the Alexander von Humboldt-Foundation while the author was in residence at Freiburg University, Germany. 
method for solving hyperbolic conservation laws containing only first order spatial derivatives, e.g. Reed and Hill [17] for solving linear equations, and Cockburn et al. $[5,4,3,6]$ for solving nonlinear equations. It is difficult to apply the DG method directly to the equations with higher order derivatives. The LDG method is an extension of the DG method aimed at solving partial differential equations (PDEs) containing higher than first order spatial derivatives. The first LDG method was constructed by Cockburn and Shu in [7] for solving nonlinear convection diffusion equations containing second order spatial derivatives. Their work was motivated by the successful numerical experiments of Bassi and Rebay [1] for the compressible Navier-Stokes equations. The idea of the LDG method is to rewrite the equations with higher order derivatives into a first order system, then apply the DG method on the system. The design of the numerical fluxes is the key ingredient to ensure stability. The LDG techniques have been developed for convection diffusion equations (containing second derivatives) [7], nonlinear one-dimensional and two-dimensional $\mathrm{KdV}$ type equations [25, 22] and Cahn-Hilliard equations [20, 21]. Recently, there is a review paper on the LDG methods for high-order time-dependent partial differential equations [24]. More general information about DG methods for elliptic, parabolic and hyperbolic partial differential equations can be found in the three special journal issues devoted to the DG method $[9,10,11]$, as well as in the recent books and lecture notes $[15,14,18,19]$.

The paper is organized as follows. In Section 2, we give some notations, definition and projections. In Section 3, we show LDG scheme for the Willmore flow of graphs and the main results in this paper. In section 4 , we give some auxiliary results which is important for our analysis. In section 5 , we present the proof of the error estimates. Concluding remarks are given in Section 6. Some of the more technical proofs of several lemmas are collected in Appendix A.

\section{Notations, definitions and projections}

We first introduce notations, definitions and projections to be used later in the paper. We define some projections and present certain interpolation and inverse properties for the finite element spaces that will be used in the error analysis.

2.1. Tessellation and function spaces. Let $\mathcal{T}_{h}$ denote a tessellation of $\Omega$ with shape-regular elements $K$. Let $\Gamma$ denote the union of the boundary faces of elements $K \in \mathcal{T}_{h}$, i.e. $\Gamma=\cup_{K \in \mathcal{T}_{h}} \partial K$, and $\Gamma_{0}=\Gamma \backslash \partial \Omega$.

In order to describe the flux functions we need to introduce some notations. Let $e$ be a face shared by the "left" and "right" elements $K_{L}$ and $K_{R}$ (we refer to [25] and [24] for a proper definition of "left" and "right" in our context). Define the normal vectors $\nu_{L}$ and $\nu_{R}$ on $e$ pointing exterior to $K_{L}$ and $K_{R}$, respectively. If $\psi$ is a function on $K_{L}$ and $K_{R}$, but possibly discontinuous across $e$, let $\psi_{L}$ denote $\left.\left(\left.\psi\right|_{K_{L}}\right)\right|_{e}$ and $\psi_{R}$ denote $\left.\left(\left.\psi\right|_{K_{R}}\right)\right|_{e}$, the left and right trace, respectively.

Let $\mathcal{Q}^{k}(K)$ be the space of tensor product of polynomials of degree at most $k \geq 0$ on $K \in \mathcal{T}_{h}$ in each variable. The finite element spaces are denoted by

$$
\begin{aligned}
V_{h} & =\left\{\varphi \in L^{2}(\Omega):\left.\quad \varphi\right|_{K} \in \mathcal{Q}^{k}(K), \quad \forall K \in \mathcal{T}_{h}\right\}, \\
\Sigma_{h} & =\left\{\boldsymbol{\eta}=\left(\eta_{1}, \cdots, \eta_{d}\right)^{T} \in\left(L^{2}(\Omega)\right)^{d}:\left.\quad \eta_{l}\right|_{K} \in \mathcal{Q}^{k}(K), \quad l=1 \cdots d, \quad \forall K \in \mathcal{T}_{h}\right\} .
\end{aligned}
$$

For one-dimensional case, we have $\mathcal{Q}^{k}(K)=\mathcal{P}^{k}(K)$ which is the space of polynomials of degree at most $k \geq 0$ defined on $K$. Note that functions in $V_{h}$ and $\Sigma_{h}$ are allowed to have discontinuities across element interfaces. Here we only consider periodic boundary conditions. Notice that the assumption of periodic boundary 
conditions is for simplicity only and not essential: the method can be easily designed for non-periodic boundary conditions. The development of the LDG method for the non-periodic boundary conditions can be found in [16].

2.2. Notations for different constants. We will adopt the following convention for different constants. These constants may have a different value in each occurrence.

We will denote by $C$ a positive constant independent of $h$, which may depend on the solution of the problem considered in this paper. For problems considered in this section, the exact solution is assumed to be smooth with periodic. Also, $0 \leq t \leq T$ for a fixed $T$. Therefore, the exact solution is always bounded.

\subsection{Projection and interpolation properties.}

2.3.1. One-dimensional case. In what follows, we will consider the standard $L^{2}$-projection of a function $\omega$ with $k+1$ continuous derivatives into space $V_{h}$,

$$
P^{ \pm}: H^{1}(\Omega) \longrightarrow V_{h},
$$

which are defined as the following. Given a function $\eta \in H^{1}(\Omega)$ and an arbitrary subinterval $K_{j}=\left(x_{j-1}, x_{j}\right)$, the restriction of $P^{ \pm} \eta$ to $K_{j}$ are defined as the elements of $\mathcal{P}^{k}\left(K_{j}\right)$ that satisfy

$$
\begin{aligned}
& \int_{K_{j}}\left(P^{+} \eta-\eta\right) w d x=0, \quad \forall w \in \mathcal{P}^{k-1}\left(K_{j}\right), \quad \text { and } \quad P^{+} \eta\left(x_{j-1}\right)=\eta\left(x_{j-1}\right), \\
& \int_{K_{j}}\left(P^{-} \eta-\eta\right) w d x=0, \quad \forall w \in \mathcal{P}^{k-1}\left(K_{j}\right), \quad \text { and } \quad P^{-} \eta\left(x_{j}\right)=\eta\left(x_{j}\right) .
\end{aligned}
$$

For the projections mentioned above, it is easy to show (c.f. [2])

$$
\left\|\eta^{e}\right\|_{\Omega}+h\left\|\eta^{e}\right\|_{L^{\infty}(\Omega)}+h^{\frac{1}{2}}\left\|\eta^{e}\right\|_{\Gamma} \leq C h^{k+1}
$$

where $\eta^{e}=\pi \eta-\eta$ or $\eta^{e}=P^{ \pm} \eta-\eta$. $\pi$ is the standard $L^{2}$ projection of the function $\eta$. The positive constant $C$, only depending on $\eta$, is independent of $h$. Here and below an unmarked norm $\|\cdot\|_{\Omega},\|\cdot\|_{\Gamma}$ refers to the usual $L^{2}$ norm for the space variables on the domain $\Omega$ and the boundary $\Gamma$.

2.3.2. Two-dimensional case. To prove the error estimates for two-dimensional problems in Cartesian meshes, we need a suitable projection $P^{ \pm}$similar to the one-dimensional case. The projections $P^{-}$for scalar functions are defined as

$$
P^{-}=P_{x}^{-} \otimes P_{y}^{-},
$$

where the subscripts $x$ and $y$ indicate that the one-dimensional projections defined by $(2.2)$ on a two-dimensional rectangle element $I \otimes J=\left[x_{j-1}, x_{j}\right] \times\left[y_{j-1}, y_{j}\right]$.

The projection $\Pi^{+}$for vector-valued function $\boldsymbol{\rho}=\left(\rho_{1}(x, y), \rho_{2}(x, y)\right)$ are defined as

$$
\Pi^{+} \boldsymbol{\rho}=\left(P_{x}^{+} \otimes \pi_{y} \rho_{1}, \pi_{x} \otimes P_{y}^{+} \rho_{2}\right) .
$$

Here $\pi_{x}, \pi_{y}$ is the standard $L^{2}$ projection in $x$ or $y$ direction. It is easy to see that, for any $\boldsymbol{\rho} \in\left[H^{1}(\Omega)\right]^{2}$, the restriction of $\Pi^{+} \boldsymbol{\rho}$ to $I \otimes J$ are elements of $\left[\mathcal{Q}^{k}(I \otimes J)\right]^{2}$ that satisfy

$$
\int_{I} \int_{J}\left(\Pi^{+} \boldsymbol{\rho}-\boldsymbol{\rho}\right) \cdot \nabla w d y d x=0
$$

for any $w \in \mathcal{Q}^{k}(I \otimes J)$, and

$$
\int_{J}\left(\Pi^{+} \boldsymbol{\rho}\left(x_{i-1}, y\right)-\boldsymbol{\rho}\left(x_{i-1}, y\right)\right) \cdot \boldsymbol{\nu} w\left(x_{i-1}^{+}, y\right) d y=0 \quad \forall w \in \mathcal{Q}^{k}(I \otimes J),
$$




$$
\int_{I}\left(\Pi^{+} \boldsymbol{\rho}\left(x, y_{j-1}\right)-\boldsymbol{\rho}\left(x, y_{j-1}\right)\right) \cdot \boldsymbol{\nu} w\left(x, y_{j-1}^{+}\right) d y=0 \quad \forall w \in \mathcal{Q}^{k}(I \otimes J),
$$

where $\boldsymbol{\nu}$ is the normal vector of the domain integrated. For the definition of similar projection on three-dimensional case, we refer to [8].

Similar to the one-dimensional case, there are some approximation results for the projections (2.4) and (2.5) in [13]

$$
\begin{array}{r}
\left\|\eta^{e}\right\|_{\Omega}+h^{\frac{1}{2}}\left\|\eta^{e}\right\|_{\Gamma} \leq C h^{k+1}\|\eta\|_{H^{k+1}(\Omega)}, \quad \forall \eta \in H^{k+1}(\Omega), \\
\left\|\boldsymbol{\rho}^{e}\right\|_{\Omega}+h^{\frac{1}{2}}\left\|\boldsymbol{\rho}^{e}\right\|_{\Gamma} \leq C h^{k+1}\|\boldsymbol{\rho}\|_{H^{k+1}(\Omega)}, \quad \forall \boldsymbol{\rho} \in\left[H^{k+1}(\Omega)\right]^{d},
\end{array}
$$

where $\eta^{e}=\pi \eta-\eta, \boldsymbol{\rho}^{e}=\pi \boldsymbol{\rho}-\boldsymbol{\rho}$ or $\eta^{e}=P^{ \pm} \eta-\eta, \boldsymbol{\rho}^{e}=\Pi^{ \pm} \boldsymbol{\rho}-\boldsymbol{\rho}$ and $C$ is independent of $h$.

The projection $P^{-}$on the Cartesian meshes has the following superconvergence property (see [13], Lemma 3.7).

Lemma 2.1. Suppose $(\eta, \boldsymbol{\rho}) \in H^{k+2}(\Omega) \otimes \Sigma_{h}$ and the projection, then we have

$$
\mid \int_{\Omega}\left(\eta-P^{-} \eta\right) \nabla \cdot \boldsymbol{\rho} d \Omega-\int_{\Gamma}\left(\eta-\widehat{\left.P^{-} \eta\right)} \boldsymbol{\rho} \cdot \boldsymbol{\nu} d \Gamma \mid \leq C h^{k+1}\|\eta\|_{H^{k+2}(\Omega)}\|\boldsymbol{\rho}\|_{L^{2}(\Omega)},\right.
$$

where "hat" term is numerical flux.

2.4. Inverse Properties and Approximation. Finally we list some inverse properties of the finite element space $V_{h}$ that will be used in our error analysis. For any $\omega_{h} \in V_{h}$, there exists a positive constant $C$ independent of $\omega_{h}$ and $h$, such that

$$
\begin{aligned}
& \text { (i) }\left\|\partial_{x} \omega_{h}\right\|_{\Omega} \leq C h^{-1}\left\|\omega_{h}\right\|_{\Omega}, \quad \text { (ii) }\left\|\omega_{h}\right\|_{\Gamma} \leq C h^{-\frac{1}{2}}\left\|\omega_{h}\right\|_{\Omega} \text {, } \\
& \text { (iii) }\left\|\omega_{h}\right\|_{L^{\infty}(\Omega)} \leq C h^{-\frac{n}{2}}\left\|\omega_{h}\right\|_{\Omega},
\end{aligned}
$$

where $d=1,2$ or 3 is the spatial dimension. For more details of these inverse properties, we refer to [2].

\section{The LDG method for the Willmore flow of graphs}

In this section, we consider the local discontinuous Galerkin method for the Willmore flow of graphs equation (1.1) in $\Omega \in \mathbb{R}^{d}$ with $d \leq 3$. We will give the energy stability property of the LDG method. The main error estimates results will be presented.

3.1. The LDG method. To define the local discontinuous Galerkin method, we rewrite equation (1.1) as a first order system:

$$
\begin{aligned}
& \frac{u_{t}}{Q}+\nabla \cdot(\boldsymbol{s}-\boldsymbol{v})=0, \\
& \boldsymbol{s}-\boldsymbol{E}(\boldsymbol{r}) \boldsymbol{p}=0, \\
& \boldsymbol{v}-\frac{1}{2} \frac{H^{2}}{Q} \boldsymbol{r}=0, \\
& \boldsymbol{p}-\nabla W=0, \\
& W-Q H=0, \\
& H-\nabla \cdot \boldsymbol{q}=0, \\
& \boldsymbol{q}-\frac{\boldsymbol{r}}{Q}=0, \\
& \boldsymbol{r}-\nabla u=0
\end{aligned}
$$


with

$$
\begin{aligned}
& \boldsymbol{E}(\boldsymbol{r})=\frac{1}{Q}\left(\boldsymbol{I}-\frac{\boldsymbol{r} \otimes \boldsymbol{r}}{Q^{2}}\right), \\
& Q=\sqrt{1+|\boldsymbol{r}|^{2}},
\end{aligned}
$$

where $\boldsymbol{s}, \boldsymbol{v}, \boldsymbol{p}, \boldsymbol{q}, \boldsymbol{r}$ are vectors, $\boldsymbol{E}(\boldsymbol{r})$ is the $d \times d$ matrix and $\boldsymbol{I}$ is the $d \times d$ identity matrix.

Applying the LDG method to the system (3.1), we have the scheme: Find $u_{h}$, $H_{h}, W_{h} \in V_{h}, \boldsymbol{s}_{h}, \boldsymbol{v}_{h}, \boldsymbol{p}_{h}, \boldsymbol{q}_{h}, \boldsymbol{r}_{h} \in \Sigma_{h}$, such that, for all test function $\varphi, \xi, \vartheta \in V_{h}$ and $\boldsymbol{\phi}, \boldsymbol{\psi}, \boldsymbol{\eta}, \boldsymbol{\rho}, \boldsymbol{\zeta} \in \Sigma_{h}$,

$$
\begin{aligned}
& \int_{K} \frac{\left(u_{h}\right)_{t}}{Q_{h}} \varphi d K-\int_{K}\left(\boldsymbol{s}_{h}-\boldsymbol{v}_{h}\right) \cdot \nabla \varphi d K+\int_{\partial K}\left(\widehat{\boldsymbol{s}_{h}} \cdot \boldsymbol{\nu}-\widehat{\boldsymbol{v}_{h}} \cdot \boldsymbol{\nu}\right) \varphi d s=0, \\
& \int_{K} \boldsymbol{s}_{h} \cdot \boldsymbol{\phi} d K-\int_{K} \boldsymbol{E}\left(\boldsymbol{r}_{h}\right) \boldsymbol{p}_{h} \cdot \boldsymbol{\phi} d K=0 \\
& \int_{K} \boldsymbol{v}_{h} \cdot \boldsymbol{\psi} d K-\int_{K} \frac{1}{2} \frac{H_{h}^{2}}{Q_{h}} \boldsymbol{r}_{h} \cdot \boldsymbol{\psi} d K=0 \\
& \int_{K} \boldsymbol{p}_{h} \cdot \boldsymbol{\eta} d K+\int_{K} W_{h} \nabla \cdot \boldsymbol{\eta} d K-\int_{\partial K} \widehat{W_{h}} \boldsymbol{\nu} \cdot \boldsymbol{\eta} d s=0, \\
& \int_{K} W_{h} \xi d K-\int_{K} Q_{h} H_{h} \xi d K=0, \\
& \int_{K} H_{h} \vartheta d K+\int_{K} \boldsymbol{q}_{h} \cdot \nabla \vartheta d K-\int_{\partial K} \widehat{\boldsymbol{q}_{h}} \cdot \boldsymbol{\nu} \vartheta d s=0 \\
& \int_{K} \boldsymbol{q}_{h} \cdot \boldsymbol{\rho} d K-\int_{K} \frac{\boldsymbol{r}_{h}}{Q_{h}} \cdot \boldsymbol{\rho} d K=0, \\
& \int_{K} \boldsymbol{r}_{h} \cdot \boldsymbol{\zeta} d K+\int_{K} u_{h} \nabla \cdot \boldsymbol{\zeta} d K-\int_{\partial K} \widehat{u_{h}} \boldsymbol{\nu} \cdot \boldsymbol{\zeta} d s=0
\end{aligned}
$$

where $\boldsymbol{\nu}$ is the normal vector to $\partial K . \boldsymbol{E}\left(\boldsymbol{r}_{h}\right)$ and $Q_{h}$ are similarly defined as follows:

$$
\begin{aligned}
& \boldsymbol{E}\left(\boldsymbol{r}_{h}\right)=\frac{1}{Q_{h}}\left(\boldsymbol{I}-\frac{\boldsymbol{r}_{h} \otimes \boldsymbol{r}_{h}}{Q_{h}^{2}}\right), \\
& Q_{h}=\sqrt{1+\left|\boldsymbol{r}_{h}\right|^{2}} .
\end{aligned}
$$

The "hat" terms in (3.4) at the cell boundary obtained after integration by parts are the so-called "numerical fluxes", which are functions defined on the cell edges and should be designed based on different guiding principles for different PDEs to ensure stability. It turns out that we can take the simple choices

$$
\left.\widehat{\boldsymbol{s}}_{h}\right|_{e}=\boldsymbol{s}_{h, R},\left.\quad \widehat{\boldsymbol{v}}_{h}\right|_{e}=\boldsymbol{v}_{h, R},\left.\quad \widehat{\boldsymbol{q}}_{h}\right|_{e}=\boldsymbol{q}_{h, R},\left.\quad \widehat{W}_{h}\right|_{e}=W_{h, L},\left.\quad \widehat{u}_{h}\right|_{e}=u_{h, L},
$$

which ensure energy stability. Numerical examples for the schemes (3.4)-(3.7) can be found in [23].

The LDG method for the Willmore flow equation satisfies the following energy stability.

Proposition 3.1. (Energy stability [23]) The solution of the Willmore flow equation using the schemes (3.4)-(3.7) satisfies energy stability

$$
\frac{1}{2} \frac{\mathrm{d}}{\mathrm{d} t} \int_{\Omega} H_{h}^{2} Q_{h} d \Omega+\int_{\Omega} \frac{\left(\left(u_{h}\right)_{t}\right)^{2}}{Q_{h}} d \Omega=0, \quad \forall u_{h}, H_{h} \in V_{h} .
$$


3.2. The main results of error estimates. In this section, we state the main error estimates of the semi-discrete LDG scheme (3.26) in Cartesian meshes.

We introduce notations

$$
\begin{aligned}
& \boldsymbol{e}_{u}=u-u_{h}, \boldsymbol{e}_{H}=H-H_{h}, \boldsymbol{e}_{W}=W-W_{h}, \boldsymbol{e}_{\boldsymbol{r}}=\boldsymbol{r}-\boldsymbol{r}_{h}, \\
& \boldsymbol{e}_{\boldsymbol{q}}=\boldsymbol{q}-\boldsymbol{q}_{h}, \boldsymbol{e}_{\boldsymbol{p}}=\boldsymbol{p}-\boldsymbol{p}_{h}, \boldsymbol{e}_{\boldsymbol{s}}=\boldsymbol{s}-\boldsymbol{s}_{h}, \boldsymbol{e}_{\boldsymbol{v}}=\boldsymbol{v}-\boldsymbol{v}_{h} .
\end{aligned}
$$

We assume the periodic boundary conditions and the equation has a unique solution $u$, which satisfy

$$
\begin{aligned}
& u \in L^{\infty}\left((0, T) ; W^{3, \infty}(\Omega)\right) \cap L^{2}\left((0, T) ; H^{4}(\Omega)\right) \cap L^{\infty}\left((0, T) ; H^{k+4}(\Omega)\right), \\
& u_{t} \in L^{\infty}\left((0, T) ; W^{2, \infty}(\Omega)\right) \cap L^{2}\left((0, T) ; L^{2}(\Omega)\right) \cap L^{\infty}\left((0, T) ; H^{k+4}(\Omega)\right),
\end{aligned}
$$

which implies $\|u\|_{L^{\infty}\left((0, T), H^{k+4}(\Omega)\right)},\left\|u_{t}\right\|_{L^{\infty}\left((0, T), H^{k+4}(\Omega)\right)},\|\boldsymbol{r}\|_{\infty},\left\|\boldsymbol{r}_{t}\right\|_{\infty},\|H\|_{\infty}$, $\left\|H_{t}\right\|_{\infty},\|\boldsymbol{p}\|_{\infty},\left\|u_{t}\right\|_{\infty}$ are all bounded. $\|\cdot\|_{\infty}$ denotes $\|\cdot\|_{L^{\infty}\left((0, T) ; L^{\infty}(\Omega)\right)}$.

Theorem 3.2. Assume that (3.1a)-(3.1h) with periodic boundary conditions has a unique solution $u$, which satisfies (3.9)-(3.10). Let $u_{h}$ be the numerical solution of the semi-discrete LDG scheme (3.4)-(3.7). For rectangular triangulation of $\Omega$, if the finite element space is the piecewise tensor product polynomials of degree $k \geq 1$, then for small enough $h$ there holds the following error estimates

$$
\begin{array}{r}
\max _{t}\left\|\boldsymbol{e}_{\boldsymbol{r}}\right\|_{\Omega}+\max _{t}\left\|\boldsymbol{e}_{H}\right\|_{\Omega} \leq C h^{k+1}, \\
\max _{t}\left\|\boldsymbol{e}_{\boldsymbol{q}}\right\|_{\Omega}+\max _{t}\left\|\boldsymbol{e}_{W}\right\|_{\Omega}+\max _{t}\left\|\boldsymbol{e}_{\boldsymbol{v}}\right\|_{\Omega} \leq C h^{k+1}, \\
\int_{0}^{T}\left\|\boldsymbol{e}_{u_{t}}\right\|_{\Omega}^{2} d t+\int_{0}^{T}\left\|\boldsymbol{e}_{\boldsymbol{p}}\right\|_{\Omega}^{2} d t+\int_{0}^{T}\left\|e_{\boldsymbol{s}}\right\|_{\Omega}^{2} d t, \leq C h^{2 k+2}, \\
\max _{t}\left\|\boldsymbol{e}_{u}\right\|_{\Omega} \leq C h^{k+1},
\end{array}
$$

where $C$ depends on $\|u\|_{L^{\infty}\left((0, T) ; H^{k+4}(\Omega)\right)},\left\|u_{t}\right\|_{L^{\infty}\left((0, T) ; H^{k+4}(\Omega)\right)},\|\boldsymbol{r}\|_{\infty},\left\|\boldsymbol{r}_{t}\right\|_{\infty}$, $\|H\|_{\infty},\left\|H_{t}\right\|_{\infty},\|\boldsymbol{p}\|_{\infty},\left\|u_{t}\right\|_{\infty}, T$.

\section{Auxiliary results}

In this section, we present some basic geometric formulas and auxiliary results which are used for error analysis.

4.1. Basic geometric formulas. We start by introducing the following notation:

$$
\begin{array}{r}
\gamma=\frac{(-\boldsymbol{r}, 1)^{T}}{Q}, \quad \gamma_{h}=\frac{\left(-\boldsymbol{r}_{h}, 1\right)^{T}}{Q_{h}}, \\
N_{h}^{K}(t)=\int_{K}\left|\boldsymbol{\gamma}-\boldsymbol{\gamma}_{h}\right|^{2} Q_{h} d K .
\end{array}
$$

Here, $\boldsymbol{r}_{h}$ is finite element approximation to $\boldsymbol{r}$. And we denote

$$
Q_{h}:=\sqrt{1+\left|\boldsymbol{r}_{h}\right|^{2}}, \quad N_{h}(t):=\sum_{K} N_{h}^{K}(t) .
$$

Here $|\cdot|$ is used for 2 -norm of a vector or 2-norm of a matrix depending on the situation.

Lemma 4.1. Using the notation introduced above, the follow inequalities hold:

$$
\begin{aligned}
& \left|\frac{1}{Q}-\frac{1}{Q_{h}}\right| \leq\left|\gamma-\gamma_{h}\right|, \quad\left|Q-Q_{h}\right| \leq Q Q_{h}\left|\gamma-\gamma_{h}\right|, \\
& \left|\frac{\boldsymbol{r}}{Q}-\frac{\boldsymbol{r}_{h}}{Q_{h}}\right| \leq\left|\boldsymbol{\gamma}-\boldsymbol{\gamma}_{h}\right|, \quad\left|\frac{\boldsymbol{r} \otimes \boldsymbol{r}}{Q}-\frac{\boldsymbol{r}_{h} \otimes \boldsymbol{r}_{h}}{Q_{h}}\right| \leq 3 Q Q_{h}\left|\boldsymbol{\gamma}-\boldsymbol{\gamma}_{h}\right|, \\
& \left|\boldsymbol{\gamma}-\boldsymbol{\gamma}_{h}\right| \leq\left|\boldsymbol{r}-\boldsymbol{r}_{h}\right| .
\end{aligned}
$$


Proof. The proof of this lemma will be given in Appendix A.1.

4.2. A priori assumption. To derive the error estimates. We need to make a priori assumption:

- $d \leq 3$

$$
\begin{gathered}
\left\|H-H_{h}\right\|_{\Omega} \leq h^{\frac{7}{4}}, \\
\left\|\boldsymbol{r}-\boldsymbol{r}_{h}\right\|_{\Omega} \leq h^{\frac{7}{4}}
\end{gathered}
$$

Then we get

$$
\begin{aligned}
\left\|\boldsymbol{r}-\boldsymbol{r}_{h}\right\|_{\infty} & \leq C h^{\frac{1}{4}}, \\
\left\|H-H_{h}\right\|_{\infty} & \leq C h^{\frac{1}{4}},
\end{aligned}
$$

where $C$ is a constant independent of $h$. So we get

$$
\left\|H_{h}\right\|_{\infty} \leq C
$$

where $C$ depends on $\|H\|_{\infty}$ and $T$.

Recalling that $Q_{h}=\sqrt{1+\left|\boldsymbol{r}_{h}\right|^{2}}$, we immediately get

$$
\left\|Q_{h}\right\|_{\infty}=\left\|\sqrt{1+\left|\boldsymbol{r}_{h}\right|^{2}}\right\|_{\infty} \leq R,
$$

where $R$ depends on $\|\boldsymbol{r}\|_{\infty}$ and $T$. Without loss of generality, let us assume $\|\boldsymbol{r}\|_{\infty}<$ $R$ and take $R=\max \left\{R,\|\boldsymbol{r}\|_{\infty}\right\}$ otherwise. This assumption will be used to get the Auxiliary Estimates Lemmas in Section 5.

Remark 4.1. The assumption will be satisfied if $k \geq 1$. We will give the explanation in the end of the proof.

4.3. Properties of matrix $\boldsymbol{E}(\boldsymbol{r})$. The matrix $\boldsymbol{E}(\boldsymbol{r})$ has the Lipschitz continuity and coercivity. We have the following properties of $\boldsymbol{E}(\boldsymbol{r})$ [12].

Lemma 4.2.

$$
\begin{aligned}
& |\boldsymbol{E}(\boldsymbol{q})-\boldsymbol{E}(\boldsymbol{p})| \leq c|\boldsymbol{q}-\boldsymbol{p}|, \\
& |\boldsymbol{E}(\boldsymbol{p})| \leq 2, \quad \boldsymbol{E}(\boldsymbol{p}) \boldsymbol{q} \cdot \boldsymbol{q} \geq \frac{|\boldsymbol{q}|^{2}}{{\sqrt{1+|\boldsymbol{p}|^{2}}}^{3}}, \quad \forall \boldsymbol{p}, \boldsymbol{q} \in R^{d} .
\end{aligned}
$$

Proof. The proof of this lemma will be given in Appendix A.2.

\section{Proof of the main result}

In this section we will give the proof of the main results. We present some auxiliary lemmas which are very crucial to our estimates. Finally, with the help of these lemmas, we obtain the error estimates.

5.1. Error equations. The numerical solutions satisfy the LDG scheme (3.4a)(3.4h). Obviously the exact solutions of the equation (3.1) also satisfy (3.4a)-(3.4h). Differentiating (3.4f)-(3.4h) with respect to time $t$ and using the relations such that

$$
\begin{aligned}
& (Q)_{t}=\left(\sqrt{1+|\boldsymbol{r}|^{2}}\right)_{t}=\frac{\boldsymbol{r} \cdot \boldsymbol{r}_{t}}{Q}, \quad\left(\frac{\boldsymbol{r}}{Q}\right)_{t}=\frac{\boldsymbol{r}_{t}}{Q}-\frac{\boldsymbol{r} \boldsymbol{r} \cdot \boldsymbol{r}_{t}}{Q^{3}}=\boldsymbol{E}(\boldsymbol{r}) \boldsymbol{r}_{t}, \\
& \left(Q_{h}\right)_{t}=\left(\sqrt{1+\left|\boldsymbol{r}_{h}\right|^{2}}\right)_{t}=\frac{\boldsymbol{r}_{h} \cdot\left(\boldsymbol{r}_{h}\right)_{t}}{Q_{h}}, \quad\left(\frac{\boldsymbol{r}_{h}}{Q_{h}}\right)_{t}=\frac{\left(\boldsymbol{r}_{h}\right)_{t}}{Q_{h}}-\frac{\boldsymbol{r}_{h} \boldsymbol{r}_{h} \cdot\left(\boldsymbol{r}_{h}\right)_{t}}{Q_{h}^{3}}=\boldsymbol{E}\left(\boldsymbol{r}_{h}\right)\left(\boldsymbol{r}_{h}\right)_{t},
\end{aligned}
$$

we combine them with (3.4a)-(3.4e) to get the error equations

$$
\int_{K}\left(\frac{u_{t}}{Q}-\frac{\left(u_{h}\right)_{t}}{Q_{h}}\right) \varphi d K-\int_{K}\left((s-\boldsymbol{v})-\left(s_{h}-\boldsymbol{v}_{h}\right)\right) \cdot \nabla \varphi d K
$$




$$
+\int_{\partial K}\left(\left(\widehat{\boldsymbol{s - s _ { h }}}\right) \cdot \boldsymbol{\nu}-\left(\widehat{\boldsymbol{v}-\boldsymbol{v}_{h}}\right) \cdot \boldsymbol{\nu}\right) \varphi d s=0
$$

$$
\int_{K}\left(\boldsymbol{s}-\boldsymbol{s}_{h}\right) \cdot \boldsymbol{\phi} d K-\int_{K}\left(\boldsymbol{E}(\boldsymbol{r}) \boldsymbol{p}-\boldsymbol{E}\left(\boldsymbol{r}_{h}\right) \boldsymbol{p}_{h}\right) \cdot \boldsymbol{\phi} d K=0,
$$

$$
\int_{K}\left(\boldsymbol{v}-\boldsymbol{v}_{h}\right) \cdot \boldsymbol{\psi} d K-\int_{K} \frac{1}{2}\left(\frac{H^{2}}{Q} \boldsymbol{r}-\frac{H_{h}^{2}}{Q_{h}} \boldsymbol{r}_{h}\right) \cdot \boldsymbol{\psi} d K=0,
$$

$$
\int_{K}\left(\boldsymbol{p}-\boldsymbol{p}_{h}\right) \cdot \boldsymbol{\eta} d K+\int_{K}\left(W-W_{h}\right) \nabla \cdot \boldsymbol{\eta} d K-\int_{\partial K}\left(\widehat{W-W_{h}}\right) \boldsymbol{\nu} \cdot \boldsymbol{\eta} d s=0,
$$

$$
\int_{K}\left(W-W_{h}\right) \xi d K-\int_{K}\left(Q H-Q_{h} H_{h}\right) \xi d K=0,
$$

$$
\left.\int_{K}\left(H_{t}-\left(H_{h}\right)_{t}\right) \vartheta d K+\int_{K}\left(\boldsymbol{q}_{t}-\left(\boldsymbol{q}_{h}\right)_{t}\right) \cdot \nabla \vartheta d K-\int_{\partial K}\left(\boldsymbol{q}_{t} \widehat{-\left(\boldsymbol{q}_{h}\right.}\right)_{t}\right) \cdot \boldsymbol{\nu} \vartheta d s=0,
$$

$$
\int_{K}\left(\boldsymbol{q}_{t}-\left(\boldsymbol{q}_{h}\right)_{t}\right) \cdot \boldsymbol{\rho} d K-\int_{K}\left(\boldsymbol{E}(\boldsymbol{r}) \boldsymbol{r}_{t}-\boldsymbol{E}\left(\boldsymbol{r}_{h}\right)\left(\boldsymbol{r}_{h}\right)_{t}\right) \cdot \boldsymbol{\rho} d K=0,
$$

$$
\int_{K}\left(\boldsymbol{r}_{t}-\left(\boldsymbol{r}_{h}\right)_{t}\right) \cdot \boldsymbol{\zeta} d K+\int_{K}\left(u_{t}-u_{h t)} \nabla \cdot \boldsymbol{\zeta} d K-\int_{\partial K}\left(u_{t} \widehat{-\left(u_{h}\right.}\right)_{t}\right) \boldsymbol{\nu} \cdot \boldsymbol{\zeta} d s=0 .
$$

Denote

$$
\begin{aligned}
& \boldsymbol{e}_{u}=u-u_{h}=u-P u+P u-u_{h}=u-P u+P \boldsymbol{e}_{u}, \\
& \boldsymbol{e}_{H}=H-H_{h}=H-P H+P H-H_{h}=H-P H+P \boldsymbol{e}_{H}, \\
& \boldsymbol{e}_{W}=W-W_{h}=W-P W+P W-W_{h}=W-P W+P \boldsymbol{e}_{W}, \\
& \boldsymbol{e}_{\boldsymbol{r}}=\boldsymbol{r}-\boldsymbol{r}_{h}=\boldsymbol{r}-\Pi \boldsymbol{r}+\Pi \boldsymbol{r}-\boldsymbol{r}_{h}=\boldsymbol{r}-\Pi \boldsymbol{r}+\Pi \boldsymbol{e}_{\boldsymbol{r}}, \\
& \boldsymbol{e}_{\boldsymbol{q}}=\boldsymbol{q}-\boldsymbol{q}_{h}=\boldsymbol{q}-\Pi \boldsymbol{q}+\Pi \boldsymbol{q}-\boldsymbol{q}_{h}=\boldsymbol{q}-\Pi \boldsymbol{q}+\Pi \boldsymbol{e}_{\boldsymbol{q}}, \\
& \boldsymbol{e}_{\boldsymbol{p}}=\boldsymbol{p}-\boldsymbol{p}_{h}=\boldsymbol{p}-\Pi \boldsymbol{p}+\Pi \boldsymbol{p}-\boldsymbol{p}_{h}=\boldsymbol{p}-\Pi \boldsymbol{p}+\Pi \boldsymbol{e}_{\boldsymbol{p}}, \\
& \boldsymbol{e}_{\boldsymbol{s}}=\boldsymbol{s}-\boldsymbol{s}_{h}=\boldsymbol{s}-\Pi \boldsymbol{s}+\Pi \boldsymbol{s}-\boldsymbol{s}_{h}=\boldsymbol{s}-\Pi \boldsymbol{s}+\Pi \boldsymbol{e}_{\boldsymbol{s}}, \\
& \boldsymbol{e}_{\boldsymbol{v}}=\boldsymbol{v}-\boldsymbol{v}_{h}=\boldsymbol{v}-\Pi \boldsymbol{v}+\Pi \boldsymbol{v}-\boldsymbol{v}_{h}=\boldsymbol{v}-\Pi \boldsymbol{v}+\Pi \boldsymbol{e}_{\boldsymbol{v}},
\end{aligned}
$$

where $P$ and $\Pi$ be the projections onto the finite element spaces $V_{h}$ and $\sum_{h}$, respectively. We choose the projection as follows

$$
\begin{aligned}
& (P, \Pi)=\left(P^{-}, P^{+}\right) \quad \text { in one dimension, } \\
& (P, \Pi)=\left(P^{-}, \Pi^{+}\right) \quad \text { in multi-dimension. }
\end{aligned}
$$

We will choose the initial data $u_{h}(x, 0)$ which can satisfy following estimates

\section{Lemma 5.1.}

$$
\begin{aligned}
& \left\|\boldsymbol{q}(x, 0)-\boldsymbol{q}_{h}(x, 0)\right\|_{\Omega}+\left\|\boldsymbol{r}(x, 0)-\boldsymbol{r}_{h}(x, 0)\right\|_{\Omega} \leq C h^{k+1}, \\
& \left\|H(x, 0)-H_{h}(x, 0)\right\|_{\Omega}+\left\|u(x, 0)-u_{h}(x, 0)\right\|_{\Omega} \leq C h^{k+1},
\end{aligned}
$$

where $C$ is some positive constant depends on the $\|u(x, 0)\|_{H^{k+3}(\Omega)}$.

Choice of the initial data $u_{h}(x, 0)$ and the proof of Lemma 5.1 will be given in Appendix A.3. 
Choosing the test functions

$$
\begin{aligned}
& \varphi=P e_{u_{t}}, \quad \phi=\Pi e_{\boldsymbol{r}_{t}}, \quad \boldsymbol{\psi}=-\Pi \boldsymbol{e}_{\boldsymbol{r}_{t}}, \quad \boldsymbol{\eta}=\Pi \boldsymbol{e}_{\boldsymbol{q}_{t}} \\
& \xi=-P \boldsymbol{e}_{H_{t}}, \quad \vartheta=P \boldsymbol{e}_{W}, \quad \boldsymbol{\rho}=-\Pi \boldsymbol{e}_{\boldsymbol{p}}, \quad \boldsymbol{\zeta}=\Pi \boldsymbol{e}_{\boldsymbol{v}}-\Pi \boldsymbol{e}_{\boldsymbol{s}}
\end{aligned}
$$

a simple calculation gives

$$
L H S=R H S
$$

where

$$
\begin{aligned}
& L H S=\int_{K}\left(\frac{u_{t}}{Q}-\frac{\left(u_{h}\right)_{t}}{Q_{h}}\right) P \boldsymbol{e}_{u_{t}} d K \\
& +\int_{K}\left(\boldsymbol{E}(\boldsymbol{r}) \boldsymbol{r}_{t}-\boldsymbol{E}\left(\boldsymbol{r}_{h}\right)\left(\boldsymbol{r}_{h}\right)_{t}\right) \cdot \Pi \boldsymbol{e}_{\boldsymbol{p}} d K-\int_{K}\left(\boldsymbol{E}(\boldsymbol{r}) \boldsymbol{p}-\boldsymbol{E}\left(\boldsymbol{r}_{h}\right) \boldsymbol{p}_{h}\right) \cdot \Pi \boldsymbol{e}_{\boldsymbol{r}_{t}} d K \\
& +\int_{K} \frac{1}{2}\left(\frac{H^{2}}{Q} \boldsymbol{r}-\frac{H_{h}^{2}}{Q_{h}} \boldsymbol{r}_{h}\right) \cdot \Pi \boldsymbol{e}_{\boldsymbol{r}_{t}}+\int_{K}\left(Q H-Q_{h} H_{h}\right) P \boldsymbol{e}_{H_{t}} d K
\end{aligned}
$$

and

$$
\begin{aligned}
& R H S=\int_{K}((\boldsymbol{s}-\Pi \boldsymbol{s})-(\boldsymbol{v}-\Pi \boldsymbol{v})) \cdot \nabla P \boldsymbol{e}_{u_{t}} d K-\int_{\partial K}((\widehat{\boldsymbol{s - \Pi} \boldsymbol{s}}) \cdot \boldsymbol{\nu}-(\widehat{\boldsymbol{v}-\Pi \boldsymbol{v}}) \cdot \boldsymbol{\nu}) P \boldsymbol{e}_{u_{t}} d s \\
& -\int_{K}(s-\Pi s) \cdot \Pi e_{\boldsymbol{r}_{t}} d K+\int_{K}(\boldsymbol{v}-\Pi \boldsymbol{v}) \cdot \Pi \boldsymbol{e}_{\boldsymbol{r}_{t}} d K-\int_{K}(\boldsymbol{p}-\Pi \boldsymbol{p}) \cdot \Pi \boldsymbol{e}_{\boldsymbol{q}_{t}} d K \\
& -\int_{K}(W-P W) \nabla \cdot \Pi \boldsymbol{e}_{\boldsymbol{q}_{t}} d K+\int_{\partial K}(\widehat{W-P} W) \boldsymbol{\nu} \cdot \Pi \boldsymbol{e}_{\boldsymbol{q}_{t}} d s+\int_{K}(W-P W) P \boldsymbol{e}_{H_{t}} d K \\
& -\int_{K}\left(H_{t}-P H_{t}\right) P \boldsymbol{e}_{W} d K-\int_{K}\left(\boldsymbol{q}_{t}-\Pi \boldsymbol{q}_{t}\right) \cdot \nabla P \boldsymbol{e}_{W} d K+\int_{\partial K}\left(\widehat{\boldsymbol{q}_{t}-\Pi \boldsymbol{q}_{t}}\right) \cdot \boldsymbol{\nu} P \boldsymbol{e}_{W} d s \\
& +\int_{K}\left(\boldsymbol{q}_{t}-\Pi \boldsymbol{q}_{t}\right) \cdot \Pi \boldsymbol{e}_{\boldsymbol{p}} d K-\int_{K}\left(\boldsymbol{r}_{t}-\Pi \boldsymbol{r}_{t}\right) \cdot\left(\Pi \boldsymbol{e}_{\boldsymbol{v}}-\Pi \boldsymbol{e}_{\boldsymbol{s}}\right) d K
\end{aligned}
$$

$$
-\int_{K}\left(u_{t}-P u_{t}\right) \nabla \cdot\left(\Pi \boldsymbol{e}_{\boldsymbol{v}}-\Pi \boldsymbol{e}_{\boldsymbol{s}}\right) d K+\int_{\partial K}\left(\widehat{u_{t}-P} u_{t}\right)\left(\Pi \boldsymbol{e}_{\boldsymbol{v}}-\Pi \boldsymbol{e}_{\boldsymbol{s}}\right) \cdot \boldsymbol{\nu} d s .
$$

For the calculation of the $L H S$, please refer to the proof for stability in [23].

5.2. Auxiliary Estimates. In this section we shall estimate some variables and nonlinear terms appeared in (5.4)

Lemma 5.2. For any time $t$, there exists $C>0$ depending on $\epsilon$ which is an any positive constant, such that

$$
\left\|P e_{u}\right\|_{\Omega}^{2} \leq \epsilon \int_{0}^{t}\left\|P e_{u_{t}}\right\|_{\Omega}^{2} d t+C \int_{0}^{t}\left\|P e_{u}\right\|_{\Omega}^{2} d t+\left\|P e_{u}(\cdot, 0)\right\|_{\Omega}^{2}
$$

Proof. We get the estimate easily by Hölder inequality

$$
\begin{aligned}
\int_{0}^{t} \frac{d}{d t}\left(\left\|P \boldsymbol{e}_{u}\right\|_{\Omega}^{2}\right) d t & =2 \int_{0}^{t} \int_{\Omega} P \boldsymbol{e}_{u} P \boldsymbol{e}_{u_{t}} d \Omega d t \\
& \leq 2 \int_{0}^{t}\left\|P \boldsymbol{e}_{u}\right\|_{\Omega}\left\|P \boldsymbol{e}_{u_{t}}\right\|_{\Omega} d t \\
& \leq \epsilon \int_{0}^{t}\left\|P \boldsymbol{e}_{u_{t}}\right\|_{\Omega}^{2} d t+\frac{1}{\epsilon} \int_{0}^{t}\left\|P e_{u}\right\|_{\Omega}^{2} d t
\end{aligned}
$$

where we use Cauchy-Schwarz inequality with $\epsilon$ in the last step for any positive constant $\epsilon>0$. 
Lemma 5.3. For any time $t$, there exists $C>0$ depends on $\|Q\|_{\infty}$ and constant $R$ defined in (4.9) such that

$$
\left\|\boldsymbol{e}_{\boldsymbol{r}}\right\|_{\Omega}^{2} \leq C N_{h}(t)
$$

Proof.

$$
\boldsymbol{e}_{\boldsymbol{r}}=\boldsymbol{r}-\boldsymbol{r}_{h}=Q\left(\frac{\boldsymbol{r}}{Q}-\frac{\boldsymbol{r}_{h}}{Q_{h}}\right)-Q\left(\frac{1}{Q}-\frac{1}{Q_{h}}\right) \boldsymbol{r}_{h}
$$

Using (4.1) and (4.2), we have

$$
\left|\boldsymbol{e}_{\boldsymbol{r}}\right| \leq Q\left|\boldsymbol{\gamma}-\boldsymbol{\gamma}_{h}\right|+Q\left|\boldsymbol{\gamma}-\boldsymbol{\gamma}_{h}\right|\left|\boldsymbol{r}_{h}\right|
$$

Thanks to a priori assumption, we have

$$
\begin{aligned}
\left|\boldsymbol{e}_{\boldsymbol{r}}\right|^{2} & \leq C\|Q\|_{\infty}^{2}\left|\gamma-\gamma_{h}\right|^{2} Q_{h}^{2} \leq C R\left|\gamma-\gamma_{h}\right|^{2} Q_{h} \\
& \leq C\left|\gamma-\gamma_{h}\right|^{2} Q_{h},
\end{aligned}
$$

where $C$ depends on $\|Q\|_{\infty}$ and constant $R$ defined in (4.9). Then we integrate both sides with spacial variable to get the estimates (5.8).

Lemma 5.4. For any time $t$, there exists $C=C\left(\|\boldsymbol{s}\|_{H^{k+1}(\Omega)},\|\boldsymbol{r}\|_{\infty},\|\boldsymbol{p}\|_{\infty}\right)>0$ such that

$$
\left\|\Pi \boldsymbol{e}_{\boldsymbol{s}}\right\|_{\Omega}^{2} \leq C\left(N_{h}(t)+h^{2 k+2}+\left\|\Pi \boldsymbol{e}_{\boldsymbol{p}}\right\|_{\Omega}^{2}\right)
$$

Proof. We consider (3.4b) separately to get the error equation

$$
\int_{K}\left(\boldsymbol{s}-\boldsymbol{s}_{h}\right) \cdot \boldsymbol{\phi} d K-\int_{K}\left(\boldsymbol{E}(\boldsymbol{r}) \boldsymbol{p}-\boldsymbol{E}\left(\boldsymbol{r}_{h}\right) \boldsymbol{p}_{h}\right) \cdot \boldsymbol{\phi}=0 .
$$

Let $\phi=\Pi e_{s}$, we have

$$
\begin{aligned}
\int_{K}\left|\Pi \boldsymbol{e}_{\boldsymbol{s}}\right|^{2} d K= & -\int_{K}(\boldsymbol{s}-\Pi \boldsymbol{s}) \cdot \Pi \boldsymbol{e}_{\boldsymbol{s}} d K \\
& +\int_{K}\left(\left(\boldsymbol{E}(\boldsymbol{r})-\boldsymbol{E}\left(\boldsymbol{r}_{h}\right)\right) \boldsymbol{p}+\boldsymbol{E}\left(\boldsymbol{r}_{h}\right)\left(\boldsymbol{p}-\boldsymbol{p}_{h}\right)\right) \cdot \Pi \boldsymbol{e}_{\boldsymbol{s}} d K .
\end{aligned}
$$

Using the Cauchy-Schwarz inequality and Lemma 4.2, we get

$$
\begin{aligned}
\int_{K}\left|\Pi \boldsymbol{e}_{\boldsymbol{s}}\right|^{2} d K & \leq \varepsilon \int_{K}\left|\Pi \boldsymbol{e}_{\boldsymbol{s}}\right|^{2} d K+\frac{1}{4 \epsilon} \int_{K}|\boldsymbol{s}-\Pi \boldsymbol{s}|^{2} d K \\
& +C \int_{K}\left|\boldsymbol{r}-\boldsymbol{r}_{h}\right|\left|\Pi \boldsymbol{e}_{\boldsymbol{s}}\right| d K+2 \int_{K}\left|\boldsymbol{p}-\boldsymbol{p}_{h}\right|\left|\Pi \boldsymbol{e}_{\boldsymbol{s}}\right| d K \\
& \leq \varepsilon \int_{K}\left|\Pi \boldsymbol{e}_{\boldsymbol{s}}\right|^{2} d K+\frac{1}{4 \epsilon} \int_{K}|\boldsymbol{s}-\Pi \boldsymbol{s}|^{2} d K \\
& +\varepsilon \int_{K}\left|\Pi \boldsymbol{e}_{\boldsymbol{s}}\right|^{2} d K+C\left(\int_{K}\left|\boldsymbol{r}-\boldsymbol{r}_{h}\right|^{2} d K+\int_{K}\left|\boldsymbol{p}-\boldsymbol{p}_{h}\right|^{2} d K\right) .
\end{aligned}
$$

Summing up all the elements $K$ and using the projection error estimates, we obtain

$$
\begin{aligned}
\left\|\Pi \boldsymbol{e}_{\boldsymbol{s}}\right\|_{\Omega}^{2} & \leq C\left(\left\|\boldsymbol{e}_{\boldsymbol{r}}\right\|_{\Omega}^{2}+h^{2 k+2}+\left\|\Pi \boldsymbol{e}_{\boldsymbol{p}}\right\|_{\Omega}^{2}\right) \\
& \leq C\left(N_{h}(t)+h^{2 k+2}+\left\|\Pi \boldsymbol{e}_{\boldsymbol{p}}\right\|_{\Omega}^{2}\right),
\end{aligned}
$$

where $C$ depends on $\|s\|_{H^{k+1}(\Omega)}$ and $\|\boldsymbol{r}\|_{\infty},\|\boldsymbol{p}\|_{\infty}$. The last step is due to Lemma 5.3 .

Lemma 5.5. For any time $t$, there exists $C=C\left(\|H\|_{\infty},\|\boldsymbol{v}\|_{H^{k+1}(\Omega)}\right)>0$, such that

$$
\left\|\Pi e_{\boldsymbol{v}}\right\|_{\Omega}^{2} \leq C\left(N_{h}(t)+h^{2 k+2}+\int_{\Omega} \boldsymbol{e}_{H}^{2} Q_{h} d \Omega\right) .
$$


Proof. We consider (3.4c) separately to get the error equation.

$$
\int_{K}\left(\boldsymbol{v}-\boldsymbol{v}_{h}\right) \cdot \boldsymbol{\psi} d K-\int_{K} \frac{1}{2}\left(\frac{H^{2}}{Q} \boldsymbol{r}-\frac{H_{h}^{2}}{Q_{h}} \boldsymbol{r}_{h}\right) \cdot \boldsymbol{\psi} d K=0 .
$$

Taking the test function $\boldsymbol{\psi}=\Pi \boldsymbol{e}_{\boldsymbol{v}}$, we have

$$
\begin{aligned}
& \int_{K}\left|\Pi \boldsymbol{e}_{\boldsymbol{v}}\right|^{2} d K \\
& =-\int_{K}(\boldsymbol{v}-\Pi \boldsymbol{v}) \cdot \Pi \boldsymbol{e}_{\boldsymbol{v}} d K+\int_{K} \frac{1}{2}\left(\frac{H^{2}}{Q} \boldsymbol{r}-\frac{H_{h}^{2}}{Q_{h}} \boldsymbol{r}_{h}\right) \cdot \Pi \boldsymbol{e}_{\boldsymbol{v}} d K \\
& =-\int_{K}(\boldsymbol{v}-\Pi \boldsymbol{v}) \cdot \Pi \boldsymbol{e}_{\boldsymbol{v}} d K+\frac{1}{2} \int_{K}\left(H^{2}\left(\frac{\boldsymbol{r}}{Q}-\frac{\boldsymbol{r}_{h}}{Q_{h}}\right)+\frac{\boldsymbol{r}_{h}}{Q_{h}}\left(H^{2}-H_{h}^{2}\right)\right) \cdot \Pi \boldsymbol{e}_{\boldsymbol{v}} d K .
\end{aligned}
$$

Using the Cauchy-Schwarz inequality, we obtain

$$
\begin{aligned}
& \int_{K}\left|\Pi \boldsymbol{e}_{\boldsymbol{v}}\right|^{2} d K \\
& \leq 3 \epsilon \int_{K}\left|\Pi \boldsymbol{e}_{\boldsymbol{v}}\right|^{2} d K+\frac{1}{4 \epsilon} \int_{K}(\boldsymbol{v}-\Pi \boldsymbol{v})^{2} d K \\
& +\frac{1}{16 \epsilon}\left(\int_{K} H^{4}\left|\frac{\boldsymbol{r}}{Q}-\frac{\boldsymbol{r}_{h}}{Q_{h}}\right|^{2} d K+\int_{K} \frac{\left|\boldsymbol{r}_{h}\right|^{2}}{Q_{h}^{2}}\left(H+H_{h}\right)^{2}\left(H-H_{h}\right)^{2} d K\right) \\
& \leq 3 \epsilon \int_{K}\left|\Pi \boldsymbol{e}_{\boldsymbol{v}}\right|^{2} d K+\frac{1}{4 \epsilon} \int_{K}(\boldsymbol{v}-\Pi \boldsymbol{v})^{2} d K \\
& +\frac{1}{16 \epsilon}\left(\|H\|_{\infty}^{4} \int_{K}\left|\frac{\boldsymbol{r}}{Q}-\frac{\boldsymbol{r}_{h}}{Q_{h}}\right|^{2} d K+\left(\|H\|_{\infty}+\left\|H_{h}\right\|_{\infty}\right)^{2} \int_{K} \frac{\left|\boldsymbol{r}_{h}\right|^{2}}{Q_{h}^{2}}\left(H-H_{h}\right)^{2} d K\right) \\
& \leq 3 \epsilon \int_{K}\left|\Pi \boldsymbol{e}_{\boldsymbol{v}}\right|^{2} d K+\frac{1}{4 \epsilon} \int_{K}(\boldsymbol{v}-\Pi \boldsymbol{v})^{2} d K \\
& +\frac{1}{16 \epsilon}\left(\|H\|_{\infty}^{4} \int_{K}\left|\boldsymbol{\gamma}-\gamma_{h}\right|^{2} Q_{h} d K+\left(\|H\|_{\infty}+\left\|H_{h}\right\|_{\infty}\right)^{2} \int_{K}\left(H-H_{h}\right)^{2} Q_{h} d K\right) .
\end{aligned}
$$

where the last step is due to Lemma 4.1 and the relations

$$
Q_{h} \geq 1, \quad Q_{h}^{2}=1+\left|\boldsymbol{r}_{h}\right|^{2}, \quad\left|\boldsymbol{r}_{h}\right|^{2} \leq Q_{h}^{2} .
$$

Adding all the elements $K$, the estimate follows by employing $L^{2}$ projection error and a priori assumption.

Lemma 5.6. For any time $t$ and every $\epsilon>0$, there exists a constant $C$, such that

$$
\left\|\boldsymbol{e}_{\boldsymbol{p}}\right\|_{\Omega}^{2} \leq \epsilon \int_{\Omega} \frac{\boldsymbol{e}_{u_{t}}^{2}}{Q_{h}} d K+C\left(N_{h}(t)+h^{2 k+2}+\int_{\Omega} \boldsymbol{e}_{H}^{2} Q_{h} d \Omega\right)
$$

with $C$ depending on $\epsilon,\|u\|_{H^{k+4}(\Omega)},\|\boldsymbol{r}\|_{\infty},\|H\|_{\infty},\left\|u_{t}\right\|_{\infty},\|\boldsymbol{p}\|_{\infty}$.

Proof. The proof of this lemma will be given in Appendix A.4.

Lemma 5.7. For any time $t$, there exists $C=C\left(\|H\|_{\infty},\|W\|_{H^{k+1}(\Omega)},\|\boldsymbol{r}\|_{\infty}\right)>0$, such that

$$
\left\|P e_{W}\right\|_{\Omega}^{2} \leq C\left(N_{h}(t)+h^{2 k+2}+\int_{\Omega} \boldsymbol{e}_{H}^{2} Q_{h} d \Omega\right) .
$$

Proof. We consider (3.4e) separately to get the error equation.

$$
\int_{K}\left(W-W_{h}\right) \xi d K-\int_{K}\left(Q H-Q_{h} H_{h}\right) \xi d K=0 .
$$


Let $\xi=P \boldsymbol{e}_{W}$, we have

$$
\begin{aligned}
\int_{K}\left(P \boldsymbol{e}_{W}\right)^{2} d K= & -\int_{K}(W-P W) P \boldsymbol{e}_{W} d K+\int_{K}\left(Q H-Q_{h} H_{h}\right) P \boldsymbol{e}_{W} d K \\
= & -\int_{K}(W-P W) P \boldsymbol{e}_{W} d K+\int_{K} H\left(\left(Q-Q_{h}\right)+\left(H-H_{h}\right) Q h\right) P \boldsymbol{e}_{W} d K \\
\leq & 3 \epsilon \int_{K}\left(P \boldsymbol{e}_{W}\right)^{2} d K+\frac{1}{4 \epsilon} \int_{K}(W-P W)^{2} d K \\
& +\frac{\|H\|_{\infty}^{2}}{4 \epsilon} \int_{K}\left(\left(Q-Q_{h}\right)^{2}+\left(H-H_{h}\right)^{2} Q_{h}^{2}\right) d K,
\end{aligned}
$$

where the last step is due to the Cauchy-Schwarz inequality. Again we use (4.1) to get

$$
\begin{aligned}
& \int_{K}\left(P \boldsymbol{e}_{W}\right)^{2} d K \\
& \leq 3 \epsilon \int_{K}\left(P \boldsymbol{e}_{W}\right)^{2} d K+\frac{1}{4 \epsilon} \int_{K}(W-P W)^{2} d K \\
& \quad+\frac{\|H\|_{\infty}^{2}}{4 \epsilon} \int_{K}\left(Q-Q_{h}\right)^{2} d K+\frac{\|H\|_{\infty}^{2}}{4 \epsilon} \int_{K}\left(H-H_{h}\right)^{2} Q_{h}^{2} d K \\
& \leq 3 \epsilon \int_{K}\left(P \boldsymbol{e}_{W}\right)^{2} d K+\frac{1}{4 \epsilon} \int_{K}(W-P W)^{2} d K \\
& \quad+\frac{\|H\|_{\infty}^{2}}{4 \epsilon} \int_{K} Q^{2} Q_{h}^{2}\left|\gamma-\gamma_{h}\right|^{2} d K+\frac{\|H\|_{\infty}^{2}}{4 \epsilon} \int_{K}\left(H-H_{h}\right)^{2} Q_{h}^{2} d K \\
& \leq 3 \epsilon \int_{K}\left(P \boldsymbol{e}_{W}\right)^{2} d K+\frac{1}{4 \epsilon} \int_{K}(W-P W)^{2} d K \\
& \quad+\frac{\|H\|_{\infty}^{2}}{4 \epsilon}\|Q\|_{\infty}^{2}\left\|Q_{h}\right\|_{\infty} \int_{K}\left|\gamma-\gamma_{h}\right|^{2} Q_{h} d K+\frac{\|H\|_{\infty}^{2}}{4 \epsilon}\left\|Q_{h}\right\|_{\infty} \int_{K}\left(H-H_{h}\right)^{2} Q_{h} d K .
\end{aligned}
$$

Taking $\epsilon=1 / 6$ and adding all elements yields (5.12). Here we use a priori assumption and the error for the projection.

Lemma 5.8. For any time $t$, there exists $C=C\left(\|\boldsymbol{q}\|_{H^{k+1}(\Omega)}\right)>0$, such that

$$
\left\|\Pi \boldsymbol{e}_{\boldsymbol{q}}\right\|_{\Omega}^{2} \leq C\left(N_{h}(t)+h^{2 k+2}\right) .
$$

Proof. We consider (3.4g) separately to get the error equation

$$
\int_{K}\left(\boldsymbol{q}-\boldsymbol{q}_{h}\right) \cdot \boldsymbol{\rho} d K-\int_{K}\left(\frac{\boldsymbol{r}}{Q}-\frac{\boldsymbol{r}_{h}}{Q_{h}}\right) \cdot \boldsymbol{\rho} d K=0,
$$

Choosing the test function $\rho=\Pi \boldsymbol{e}_{\boldsymbol{q}}$, we have

$$
\int_{K}\left|\Pi e_{\boldsymbol{q}}\right|^{2} d K=-\int_{K}(\boldsymbol{q}-\Pi \boldsymbol{q}) \cdot \Pi e_{\boldsymbol{q}} d K+\int_{K}\left(\frac{\boldsymbol{r}}{Q}-\frac{\boldsymbol{r}_{h}}{Q_{h}}\right) \cdot \Pi \boldsymbol{e}_{\boldsymbol{q}} d K,
$$

Adding all elements $K$ and using Lemma 4.1, we get

$$
\sum_{K} \int_{K}\left|\Pi \boldsymbol{e}_{\boldsymbol{q}}\right|^{2} d K \leq \epsilon \sum_{K} \int_{K}\left|\Pi \boldsymbol{e}_{\boldsymbol{q}}\right|^{2} d K+C h^{2 k+2}+\sum_{K} \int_{K}\left|\gamma-\gamma_{h}\right|\left|\Pi \boldsymbol{e}_{\boldsymbol{q}}\right| d K,
$$

where $C$ comes from the error for the projection which depends on $\|\boldsymbol{q}\|_{H^{k+1}(\Omega)}$. For any positive constant $\epsilon>0$, again we employ the Cauchy-Schwarz inequality to get

$$
\sum_{K} \int_{K}\left|\Pi \boldsymbol{e}_{\boldsymbol{q}}\right|^{2} d K \leq 2 \epsilon \sum_{K} \int_{K}\left|\Pi \boldsymbol{e}_{\boldsymbol{q}}\right|^{2} d K+C h^{2 k+2}+\frac{1}{4 \epsilon} \sum_{K} \int_{K}\left|\boldsymbol{\gamma}-\gamma_{h}\right|^{2} d K
$$




$$
\begin{aligned}
& \leq 2 \epsilon \sum_{K} \int_{K}\left|\Pi e_{\boldsymbol{q}}\right|^{2} d K+C h^{2 k+2}+\frac{1}{4 \epsilon} \sum_{K} \int_{K}\left|\gamma-\gamma_{h}\right|^{2} Q_{h} d K \\
& =2 \epsilon \sum_{K} \int_{K}\left|\Pi e_{\boldsymbol{q}}\right|^{2} d K+C h^{2 k+2}+\frac{1}{4 \epsilon} N_{h}(t) .
\end{aligned}
$$

By taking $\epsilon=1 / 4$ we get the estimate (5.13).

Lemma 5.9. For any time $t$, there exists $C=C\left(\left\|\boldsymbol{r}_{t}\right\|_{\infty}\right)>0$, such that

$$
\int_{K}\left(\frac{\boldsymbol{r}}{Q}-\frac{\boldsymbol{r}_{h}}{Q_{h}}\right) \cdot \boldsymbol{e}_{\boldsymbol{r}_{t}} d K \geq \frac{1}{2} \frac{d}{d t} N_{h}^{K}(t)-C N_{h}^{K}(t) .
$$

Proof. The proof of this lemma will be given in Appendix A.5.

Lemma 5.10. For any time $t$ and every $\epsilon>0$ there exist a positive $C$ such that

$$
\frac{d}{d t} N_{h}(t) \leq C\left(N_{h}(t)+h^{2 k+2}+\int_{\Omega} e_{H}^{2} Q_{h} d \Omega\right)+\epsilon \int_{\Omega} \frac{e_{u_{t}}^{2}}{Q_{h}} d \Omega,
$$

where $C$ depends on $\left\|u_{t}\right\|_{H^{k+1}(\Omega)},\|H\|_{H^{k+1}(\Omega)},\left\|\boldsymbol{r}_{t}\right\|_{H^{k+1}(\Omega)},\|\boldsymbol{q}\|_{H^{k+1}(\Omega)}$.

Proof. The proof of this lemma will be given in Appendix A.6.

Lemma 5.11. For any time $t$,

$$
\int_{\Omega}\left(\frac{u_{t}}{Q}-\frac{\left(u_{h}\right)_{t}}{Q_{h}}\right) P e_{u_{t}} d \Omega \geq \frac{1}{2} \int_{\Omega} \frac{\boldsymbol{e}_{u_{t}}^{2}}{Q_{h}} d \Omega-C\left(N_{h}(t)+h^{2 k+2}\right),
$$

where $C$ depends on $\left\|u_{t}\right\|_{\infty},\|\boldsymbol{r}\|_{\infty},\left\|u_{t}\right\|_{H^{k+1}(\Omega)}$.

Proof.

$$
\begin{aligned}
& \int_{K}\left(\frac{u_{t}}{Q}-\frac{\left(u_{h}\right)_{t}}{Q_{h}}\right) P \boldsymbol{e}_{u_{t}} d K \\
& =\int_{K}\left(\frac{u_{t}}{Q}-\frac{\left(u_{h}\right)_{t}}{Q_{h}}\right)\left(u_{t}-\left(u_{h}\right)_{t}\right) d K-\int_{K}\left(\frac{u_{t}}{Q}-\frac{\left(u_{h}\right)_{t}}{Q_{h}}\right)\left(u_{t}-P u_{t}\right) d K \\
& =\int_{K} \frac{e_{u_{t}}^{2}}{Q_{h}} d K+\int_{K} u_{t}\left(\frac{1}{Q}-\frac{1}{Q_{h}}\right)\left(u_{t}-\left(u_{h}\right)_{t}\right) d K \\
& \quad-\int_{K} u_{t}\left(\frac{1}{Q}-\frac{1}{Q_{h}}\right)\left(u_{t}-P u_{t}\right) d K-\int_{K} \frac{\left(u_{t}-\left(u_{h}\right)_{t}\right)\left(u_{t}-P u_{t}\right)}{Q_{h}} d K .
\end{aligned}
$$

For any positive constant $\epsilon>0$, employing Cauchy-Schwarz inequality and (4.1), we have

$$
\begin{aligned}
& \left|\sum_{K} \int_{K} u_{t}\left(\frac{1}{Q}-\frac{1}{Q_{h}}\right)\left(u_{t}-\left(u_{h}\right)_{t}\right) d K\right| \\
& =\left|\sum_{K} \int_{K} u_{t} \sqrt{Q_{h}}\left(\frac{1}{Q}-\frac{1}{Q_{h}}\right) \frac{\left(u_{t}-\left(u_{h}\right)_{t}\right)}{\sqrt{Q_{h}}} d K\right| \\
& \leq \epsilon \sum_{K} \int_{K} \frac{e_{u_{t}}^{2}}{Q_{h}} d K+\frac{\left\|u_{t}\right\|_{\infty}^{2}}{4 \epsilon} \sum_{K} \int_{K}\left(\frac{1}{Q}-\frac{1}{Q_{h}}\right)^{2} Q_{h} d K \\
& \leq \epsilon \sum_{K} \int_{K} \frac{e_{u_{t}}^{2}}{Q_{h}} d K+\frac{\left\|u_{t}\right\|_{\infty}^{2}}{4 \epsilon} \sum_{K} \int_{K}\left|\gamma-\gamma_{h}\right|^{2} Q_{h} d K \\
& =\epsilon \sum_{K} \int_{K} \frac{e_{u_{t}}^{2}}{Q_{h}} d K+\frac{\left\|u_{t}\right\|_{\infty}^{2}}{4 \epsilon} N_{h}(t) .
\end{aligned}
$$


Similarly we can estimate the other two terms

$$
\begin{aligned}
& \left|-\sum_{K} \int_{K} u_{t}\left(\frac{1}{Q}-\frac{1}{Q_{h}}\right)\left(u_{t}-P u_{t}\right) d K\right| \\
& \leq \frac{1}{2} \sum_{K} \int_{K}\left(u_{t}-P u_{t}\right)^{2}+\frac{\left\|u_{t}\right\|_{\infty}^{2}}{2} \sum_{K} \int_{K}\left(\frac{1}{Q}-\frac{1}{Q_{h}}\right)^{2} d K \\
& \leq \frac{1}{2} \sum_{K} \int_{K}\left(u_{t}-P u_{t}\right)^{2}+\frac{\left\|u_{t}\right\|_{\infty}^{2}}{2} \sum_{K} \int_{K}\left|\gamma-\gamma_{h}\right|^{2} d K \\
& \leq \frac{1}{2} \sum_{K} \int_{K}\left(u_{t}-P u_{t}\right)^{2}+\frac{\left\|u_{t}\right\|_{\infty}^{2}}{2} \sum_{K} \int_{K}\left|\gamma-\gamma_{h}\right|^{2} Q_{h} d K \quad \leq C h^{2 k+2}+\frac{\left\|u_{t}\right\|_{\infty}^{2}}{2} N_{h}(t),
\end{aligned}
$$

and

$$
\begin{aligned}
\left|-\sum_{K} \int_{K} \frac{\left(u_{t}-\left(u_{h}\right)_{t}\right)\left(u_{t}-P u_{t}\right)}{Q_{h}} d K\right| & \leq\left|-\sum_{K} \int_{K} \frac{\left(u_{t}-\left(u_{h}\right)_{t}\right)}{\sqrt{Q_{h}}} \frac{\left(u_{t}-P u_{t}\right)}{\sqrt{Q_{h}}} d K\right| \\
& \leq \epsilon \sum_{K} \int_{K} \frac{e_{u_{t}}^{2}}{Q_{h}} d K+\frac{1}{4 \epsilon} \sum_{K} \int_{K} \frac{\left(u_{t}-P u_{t}\right)^{2}}{Q_{h}} d K \\
& \leq \epsilon \sum_{K} \int_{K} \frac{e_{u_{t}}^{2}}{Q_{h}} d K+\frac{1}{4 \epsilon} C h^{2 k+2},
\end{aligned}
$$

where $C$ depends on $\left\|u_{t}\right\|_{H^{k+1}(\Omega)}, \epsilon$. Using three terms above,we get

$$
\begin{aligned}
& \sum_{k} \int_{K} u_{t}\left(\frac{1}{Q}-\frac{1}{Q_{h}}\right)\left(u_{t}-\left(u_{h}\right)_{t}\right) d K \\
& \quad-\sum_{K} \int_{K} u_{t}\left(\frac{1}{Q}-\frac{1}{Q_{h}}\right)\left(u_{t}-P u_{t}\right) d K-\sum_{K} \int_{K} \frac{\left(u_{t}-\left(u_{h}\right)_{t}\right)\left(u_{t}-P u_{t}\right)}{Q_{h}} d K \\
& \geq-2 \epsilon \sum_{K} \int_{K} \frac{e_{u_{t}}^{2}}{Q_{h}} d K-C\left(N_{h}(t)+h^{2 k+2}\right),
\end{aligned}
$$

where $C$ depends on $\left\|u_{t}\right\|_{H^{k+1}(\Omega)}$ and $\epsilon$. By taking $\epsilon=1 / 4$, we get estimates (5.16).

Lemma 5.12. For any time $t$ and every $\epsilon>0$, there exists $C>0$, such that

$$
\begin{aligned}
& \sum_{K}\left(\int_{K}\left(\boldsymbol{E}(\boldsymbol{r}) \boldsymbol{r}_{t}-\boldsymbol{E}\left(\boldsymbol{r}_{h}\right)\left(\boldsymbol{r}_{h}\right)_{t}\right) \cdot \Pi \boldsymbol{e}_{\boldsymbol{p}} d K-\int_{K}\left(\boldsymbol{E}(\boldsymbol{r}) \boldsymbol{p}-\boldsymbol{E}\left(\boldsymbol{r}_{h}\right) \boldsymbol{p}_{h}\right) \cdot \Pi \boldsymbol{e}_{\boldsymbol{r}_{t}} d K\right) \\
& \geq-\frac{d}{d t} \int_{\Omega}\left(\left(\frac{Q_{h}}{\widetilde{Q_{h}}}-1\right)\left(\widetilde{\gamma_{h}}-\gamma_{h}\right)-\frac{1}{2}\left(\frac{Q_{h}}{\widetilde{Q_{h}}} \widetilde{\gamma_{h}}-\left.\gamma_{h}\right|^{2} \widetilde{\gamma_{h}}\right)\right) \cdot(\Pi \boldsymbol{p}, 0)^{T} d \Omega \\
& -\frac{d}{d t} \sum_{K} \int_{K}(\boldsymbol{E}(\boldsymbol{r}) \boldsymbol{p}-\boldsymbol{E}(\Pi \boldsymbol{r}) \Pi \boldsymbol{p}) \cdot \Pi \boldsymbol{e}_{\boldsymbol{r}} d K \\
& -C\left(N_{h}(t)+h^{2 k+2}\right)-\epsilon\left\|\Pi \boldsymbol{e}_{\boldsymbol{p}}\right\|_{\Omega}^{2}
\end{aligned}
$$

with $C$ depending on $\epsilon,\|\boldsymbol{r}\|_{\infty},\|\boldsymbol{p}\|_{\infty},\|\boldsymbol{r}\|_{H^{k+1}(\Omega)},\left\|\boldsymbol{r}_{t}\right\|_{H^{k+1}(\Omega)},\|\boldsymbol{p}\|_{H^{k+1}(\Omega)},\left\|\boldsymbol{p}_{t}\right\|_{H^{k+1}(\Omega)}$, where

$$
\widetilde{Q_{h}}=\sqrt{1+|\Pi \boldsymbol{r}|^{2}}, \quad \widetilde{\gamma_{h}}=\frac{(-\Pi \boldsymbol{r}, 1)^{T}}{\widetilde{Q_{h}}} .
$$

Proof. The proof of this lemma will be given in Appendix A.7. 
Lemma 5.13. For any time $t$, there exists a positive $C$, such that

$$
\begin{aligned}
& \int_{\Omega}\left(Q H-Q_{h} H_{h}\right) P \boldsymbol{e}_{H_{t}} d \Omega+\int_{\Omega} \frac{1}{2}\left(\frac{H^{2}}{Q} \boldsymbol{r}-\frac{H_{h}^{2}}{Q_{h}} \boldsymbol{r}_{h}\right) \cdot \Pi \boldsymbol{e}_{\boldsymbol{r}_{t}} d \Omega \\
& \geq \frac{1}{2} \frac{d}{d t} \int_{\Omega} \boldsymbol{e}_{H}^{2} Q_{h} d \Omega+\frac{d}{d t} \int_{\Omega}\left(Q-Q_{h}\right)\left(H-H_{h}\right) H d \Omega+\int_{\Omega} \frac{1}{2} H^{2} \partial_{t}\left(\frac{1}{2}\left|\boldsymbol{\gamma}-\gamma_{h}\right|^{2} Q_{h}\right) d \Omega \\
& -C\left(N_{h}(t)+\int_{\Omega} \boldsymbol{e}_{H}^{2} d \Omega+h^{2 k+2}\right),
\end{aligned}
$$

where $C$ depends on $\|\boldsymbol{r}\|_{\infty},\|H\|_{\infty},\left\|\boldsymbol{r}_{t}\right\|_{\infty},\left\|H_{t}\right\|_{H^{k+1}(\Omega)}$.

Proof. The proof of this lemma will be given in Appendix A.8.

5.3. Proof of the estimates. We firstly estimate the right hand of (5.4). Using the projection $\Pi$ and numerical fluxes, we get

$$
\sum_{K} R H S=\mathcal{X}_{1}+\mathcal{X}_{2}+\mathcal{X}_{3}+\mathcal{X}_{4}
$$

where

$$
\begin{aligned}
& \mathcal{X}_{1}=-\int_{\Omega}(\boldsymbol{s}-\Pi \boldsymbol{s}) \cdot \Pi \boldsymbol{e}_{\boldsymbol{r}_{t}} d \Omega+\int_{\Omega}(\boldsymbol{v}-\Pi \boldsymbol{v}) \cdot \Pi \boldsymbol{e}_{\boldsymbol{r}_{t}} d \Omega-\int_{\Omega}(\boldsymbol{p}-\Pi \boldsymbol{p}) \cdot \Pi \boldsymbol{e}_{\boldsymbol{q}_{t}} d \Omega \\
&+\int_{\Omega}(W-P W) P \boldsymbol{e}_{H_{t}} d \Omega \\
& \mathcal{X}_{2}=-\int_{\Omega}\left(H_{t}-P H_{t}\right) P \boldsymbol{e}_{W} d \Omega+\int_{\Omega}\left(\boldsymbol{q}_{t}-\Pi \boldsymbol{q}_{t}\right) \cdot \Pi \boldsymbol{e}_{\boldsymbol{p}} d \Omega-\int_{K}\left(\boldsymbol{r}_{t}-\Pi \boldsymbol{r}_{t}\right) \cdot\left(\Pi \boldsymbol{e}_{\boldsymbol{v}}-\Pi \boldsymbol{e}_{s}\right), \\
& \mathcal{X}_{3}=-\int_{\Omega}\left(u_{t}-P u_{t}\right) \nabla \cdot\left(\Pi \boldsymbol{e}_{\boldsymbol{v}}-\Pi \boldsymbol{e}_{s}\right) d \Omega+\int_{\Gamma}\left(\widehat{u_{t}-P u_{t}}\right)\left(\Pi \boldsymbol{e}_{\boldsymbol{v}}-\Pi \boldsymbol{e}_{s}\right) \cdot \boldsymbol{\nu} d \Gamma \\
& \mathcal{X}_{4}=-\int_{\Omega}(W-P W) \nabla \cdot \Pi \boldsymbol{e}_{\boldsymbol{q}_{t}}+\int_{\Gamma}(\widehat{W-P} W) \boldsymbol{\nu} \cdot \Pi \boldsymbol{e}_{\boldsymbol{q}_{t}} d \Gamma
\end{aligned}
$$

Now we estimate $\mathcal{X}_{1}, \mathcal{X}_{2}, \mathcal{X}_{3}, \mathcal{X}_{4}$.

- Estimate $\mathcal{X}_{1}$.

Integrating $\mathcal{X}_{1}$ with respect to time $t$

$$
\begin{aligned}
\int_{0}^{t} \mathcal{X}_{1} d t= & -\int_{\Omega}(s-\Pi s) \cdot \Pi e_{\boldsymbol{r}} d \Omega+\int_{0}^{t} \int_{\Omega}\left(\boldsymbol{s}_{t}-\Pi \boldsymbol{s}_{t}\right) \cdot \Pi \boldsymbol{e}_{\boldsymbol{r}} d \Omega d t \\
& +\int_{\Omega}(\boldsymbol{v}-\Pi \boldsymbol{v}) \cdot \Pi \boldsymbol{e}_{\boldsymbol{r}} d \Omega-\int_{0}^{t} \int_{\Omega}\left(\boldsymbol{v}_{t}-\Pi \boldsymbol{v}_{t}\right) \cdot \Pi \boldsymbol{e}_{\boldsymbol{r}} d \Omega d t \\
& -\int_{\Omega}(\boldsymbol{p}-\Pi \boldsymbol{p}) \cdot \Pi \boldsymbol{e}_{\boldsymbol{q}} d \Omega-\int_{0}^{t} \int_{\Omega}\left(\boldsymbol{p}_{t}-\Pi \boldsymbol{p}_{t}\right) \cdot \Pi \boldsymbol{e}_{\boldsymbol{q}} d \Omega d t \\
& +\int_{\Omega}(W-P W) P \boldsymbol{e}_{H} d \Omega-\int_{0}^{t} \int_{\Omega}\left(W_{t}-P W_{t}\right) P \boldsymbol{e}_{H} d \Omega d t
\end{aligned}
$$

In view of Lemma 5.3 and Lemma 5.8, we have

$$
\left|\int_{0}^{t} \mathcal{X}_{1} d t\right| \leq \epsilon \int_{\Omega}\left(\boldsymbol{e}_{H}^{2}+\left|\Pi \boldsymbol{e}_{q}\right|^{2}+\left|\boldsymbol{e}_{\boldsymbol{r}}\right|^{2}\right) d \Omega+C\left(h^{2 k+2}+\int_{0}^{t}\left(N_{h}(t)+\int_{\Omega} \boldsymbol{e}_{H}^{2} d \Omega\right) d t\right),
$$

where $C$ depends on $\varepsilon,\|u\|_{H^{k+4}(\Omega)},\left\|u_{t}\right\|_{H^{k+4}(\Omega)}$.

- Estimate $\mathcal{X}_{2}$.

$$
\left|\mathcal{X}_{2}\right| \leq \epsilon \int_{\Omega}\left(P \boldsymbol{e}_{W}^{2}+\left|\boldsymbol{e}_{\boldsymbol{p}}\right|^{2}+\left|\boldsymbol{e}_{\boldsymbol{v}}\right|^{2}+\left|\boldsymbol{e}_{\boldsymbol{s}}\right|^{2}\right) d \Omega+C h^{2 k+2}
$$


where $C$ depends on depending on $\epsilon,\left\|u_{t}\right\|_{H^{k+3}(\Omega)}$. Using Lemma 5.4, Lemma 5.5, Lemma 5.6, Lemma 5.7

$$
\left|\mathcal{X}_{2}\right| \leq \epsilon \int_{\Omega} \frac{e_{u_{t}}^{2}}{Q_{h}} d \Omega+C\left(N_{h}(t)+\int_{\Omega} e_{H}^{2} Q_{h} d \Omega+h^{2 k+2}\right),
$$

where $C$ depends on $\epsilon,\|u\|_{H^{k+4}(\Omega)},\|\boldsymbol{r}\|_{\infty},\|H\|_{\infty},\left\|u_{t}\right\|_{\infty},\|\boldsymbol{p}\|_{\infty}$.

- Estimate $\mathcal{X}_{3}$.

In one-dimension, $\mathcal{X}_{3}=0$. In multi-dimension, recalling Lemma (2.1), we get

$$
\left|\mathcal{X}_{3}\right| \leq C h^{k+1}\left(\left\|\boldsymbol{e}_{\boldsymbol{v}}\right\|_{\Omega}^{2}+\left\|\boldsymbol{e}_{s}\right\|_{\Omega}^{2}\right),
$$

where $C$ depends on $\left\|u_{t}\right\|_{H^{k+2}}$. Employing Lemma 5.4, Lemma 5.5, Lemma 5.6 we obtain

$$
\left|\mathcal{X}_{3}\right| \leq \epsilon \int_{\Omega} \frac{e_{u_{t}}^{2}}{Q_{h}} d \Omega+C\left(N_{h}(t)+\int_{\Omega} \boldsymbol{e}_{H}^{2} Q_{h} d \Omega+h^{2 k+2}\right),
$$

where $C$ depends on $\epsilon,\|u\|_{H^{k+4}(\Omega)},\|\boldsymbol{r}\|_{\infty},\|H\|_{\infty},\left\|u_{t}\right\|_{\infty},\|\boldsymbol{p}\|_{\infty}$.

- Estimate $\mathcal{X}_{4}$.

In one-dimension, $\mathcal{X}_{4}=0$. In multi-dimension, integrating $\mathcal{X}_{4}$ with respect to time $t$

$$
\begin{aligned}
\int_{0}^{t} \mathcal{X}_{4} d t= & -\int_{\Omega}(W-P W) \nabla \cdot \Pi \boldsymbol{e}_{\boldsymbol{q}} d \Omega+\int_{\Gamma}(\widehat{W-P} W) \boldsymbol{\nu} \cdot \Pi \boldsymbol{e}_{\boldsymbol{q}} d \Gamma \\
& +\int_{0}^{t} \int_{\Omega}\left(W_{t}-P W_{t}\right) \nabla \cdot \Pi \boldsymbol{e}_{\boldsymbol{q}} d \Omega d t-\int_{0}^{t} \int_{\Gamma}\left(\widehat{W_{t}-P W_{t}}\right) \boldsymbol{\nu} \cdot \Pi \boldsymbol{e}_{\boldsymbol{q}} d \Gamma d t
\end{aligned}
$$

Using Lemma 2.1 and Lemma 5.8, we get

$$
\left|\int_{0}^{t} \mathcal{X}_{4} d t\right| \leq \epsilon N_{h}(t)+C\left(h^{2 k+2}+\int_{0}^{t} N_{h}(t) d t\right)
$$

where $C$ depends on $\|W\|_{H^{k+1}},\left\|W_{t}\right\|_{H^{k+1}},\|\boldsymbol{q}\|_{H^{k+1}}$.

Collecting the estimates $\mathcal{X}_{1}, \mathcal{X}_{2}, \mathcal{X}_{3}, \mathcal{X}_{4}$, we obtain

$$
\begin{aligned}
\left|\int_{0}^{t} \sum_{i=1}^{4}\left(\mathcal{X}_{i}\right) d t\right| & \leq \epsilon \int_{0}^{t}\left(\int_{\Omega}\left(\frac{\boldsymbol{e}_{u_{t}}^{2}}{Q_{h}}+\boldsymbol{e}_{H}^{2}\right) d \Omega+N_{h}(t)\right) d t \\
& +C \int_{0}^{t}\left(N_{h}(t)+\int_{\Omega} \boldsymbol{e}_{H}^{2} d \Omega\right) d t+C h^{2 k+2},
\end{aligned}
$$

where $C$ depends on $\varepsilon,\|u\|_{H^{k+4}(\Omega)},\left\|u_{t}\right\|_{H^{k+4}(\Omega)},\|\boldsymbol{r}\|_{\infty},\|H\|_{\infty},\left\|u_{t}\right\|_{\infty},\|\boldsymbol{p}\|_{\infty}$. Using Lemma 5.11, Lemma 5.12, Lemma 5.13, we obtain from (5.4)

$$
\begin{aligned}
& \frac{1}{2} \int_{\Omega} \frac{\boldsymbol{e}_{u_{t}}^{2}}{Q_{h}} d \Omega+\frac{1}{2} \frac{d}{d t} \int_{\Omega} \boldsymbol{e}_{H}^{2} Q_{h} d \Omega \\
& \leq-\frac{d}{d t} \int_{\Omega}\left(Q-Q_{h}\right)\left(H-H_{h}\right) H d \Omega-\int_{\Omega} \frac{1}{2} H^{2} \partial_{t}\left(\frac{1}{2}\left|\boldsymbol{\gamma}-\gamma_{h}\right|^{2} Q_{h}\right) d \Omega \\
& +\frac{d}{d t} \int_{\Omega}\left(\left(\frac{Q_{h}}{\widetilde{Q_{h}}}-1\right)\left(\widetilde{\gamma_{h}}-\gamma_{h}\right)+\frac{1}{2}\left(\frac{Q_{h}}{\widetilde{Q_{h}}}\left|\widetilde{\gamma_{h}}-\gamma_{h}\right|^{2} \widetilde{\gamma_{h}}\right)\right) \cdot(\Pi \boldsymbol{p}, 0)^{T} d \Omega \\
& +\frac{d}{d t} \sum_{K} \int_{K}(\boldsymbol{E}(\boldsymbol{r}) \boldsymbol{p}-\boldsymbol{E}(\Pi \boldsymbol{r}) \Pi \boldsymbol{p}) \cdot \Pi \boldsymbol{e}_{\boldsymbol{r}} d K+\epsilon\left\|\Pi \boldsymbol{e}_{\boldsymbol{p}}\right\|_{\Omega}^{2} \\
& +\mathcal{X}_{1}+\mathcal{X}_{2}+\mathcal{X}_{3}+\mathcal{X}_{4}+C\left(N_{h}(t)+\int_{\Omega} \boldsymbol{e}_{H}^{2} d \Omega+h^{2 k+2}\right)
\end{aligned}
$$


Denote

$$
\begin{aligned}
\mathcal{X}_{5} & =-\frac{d}{d t} \int_{\Omega}\left(Q-Q_{h}\right)\left(H-H_{h}\right) H d \Omega-\int_{\Omega} \frac{1}{2} H^{2} \partial_{t}\left(\frac{1}{2}\left|\boldsymbol{\gamma}-\gamma_{h}\right|^{2} Q_{h}\right) d \Omega \\
& +\frac{d}{d t} \int_{\Omega}\left(\left(\frac{Q_{h}}{\widetilde{Q_{h}}}-1\right)\left(\widetilde{\gamma_{h}}-\gamma_{h}\right)+\frac{1}{2}\left(\frac{Q_{h}}{\widetilde{Q_{h}}}\left|\widetilde{\gamma_{h}}-\gamma_{h}\right|^{2} \widetilde{\gamma_{h}}\right)\right) \cdot(\Pi \boldsymbol{p}, 0)^{T} d \Omega \\
& +\frac{d}{d t} \sum_{K} \int_{K}(\boldsymbol{E}(\boldsymbol{r}) \boldsymbol{p}-\boldsymbol{E}(\Pi \boldsymbol{r}) \Pi \boldsymbol{p}) \cdot \Pi \boldsymbol{e}_{\boldsymbol{r}} d K \\
= & -\frac{d}{d t} \int_{\Omega}\left(Q-Q_{h}\right)\left(H-H_{h}\right) H d \Omega \\
& -\frac{d}{d t} \int_{\Omega} \frac{1}{2} H^{2}\left(\frac{1}{2}\left|\boldsymbol{\gamma}-\gamma_{h}\right|^{2} Q_{h}\right) d \Omega+\int_{\Omega} H H_{t}\left(\frac{1}{2}\left|\boldsymbol{\gamma}-\gamma_{h}\right|^{2} Q_{h}\right) d \Omega \\
& +\frac{d}{d t} \int_{\Omega}\left(\left(\frac{Q_{h}}{\widetilde{Q_{h}}}-1\right)\left(\widetilde{\gamma_{h}}-\gamma_{h}\right)+\frac{1}{2}\left(\frac{Q_{h}}{\widetilde{Q_{h}}}\left|\widetilde{\gamma_{h}}-\gamma_{h}\right|^{2} \widetilde{\gamma_{h}}\right)\right) \cdot(\Pi \boldsymbol{p}, 0)^{T} d \Omega \\
& +\frac{d}{d t} \sum_{K} \int_{K}(\boldsymbol{E}(\boldsymbol{r}) \boldsymbol{p}-\boldsymbol{E}(\Pi \boldsymbol{r}) \Pi \boldsymbol{p}) \cdot \Pi \boldsymbol{e}_{\boldsymbol{r}} d K .
\end{aligned}
$$

Integrating $\mathcal{X}_{5}$ with respect to time $t$, we have estimate

$$
\left|\int_{0}^{t} \mathcal{X}_{5} d t\right| \leq \frac{1}{4} \int_{\Omega} e_{H}^{2} Q_{h} d \Omega+C \int_{0}^{t} N_{h}(t) d t+C h^{2 k+2}+C_{1} N_{h}(t),
$$

where $C$ depends on $\|\boldsymbol{r}\|_{H^{k+1}(\Omega)},\|\boldsymbol{p}\|_{H^{k+1}(\Omega)},\left\|H_{t}\right\|_{\infty},\|H\|_{\infty}$. And $C_{1}$ depends on $\|\boldsymbol{r}\|_{\infty},\|H\|_{\infty},\|\Pi \boldsymbol{p}\|_{\infty},\left\|\widetilde{\gamma_{h}}\right\|_{\infty}$. Taking

$$
R_{0}=C_{1}+1
$$

we sum the following terms

$$
(5.15) \times R_{0}+(5.18)+(5.11) .
$$

Integrating with respect to time $t$ and choosing $\epsilon$ sufficiently small, we obtain

$$
\begin{aligned}
& \int_{0}^{t}\left(\left\|\boldsymbol{e}_{u_{t}}\right\|_{\Omega}^{2}+\left\|\boldsymbol{e}_{\boldsymbol{p}}\right\|_{\Omega}^{2}\right) d t+N_{h}(t)+\left\|\boldsymbol{e}_{H}\right\|_{\Omega}^{2} \\
& \leq C \int_{0}^{t}\left(N_{h}(t)+\left\|\boldsymbol{e}_{H}\right\|_{\Omega}^{2}\right) d t+C h^{2 k+2}
\end{aligned}
$$

where we use the error estimate for the initial date on Lemma 5.1. Gronwall inequality yields

$$
\begin{gathered}
\max _{t}\left(N_{h}(t)+\left\|\boldsymbol{e}_{H}\right\|_{\Omega}^{2}\right) \leq C h^{2 k+2}, \\
\int_{0}^{T}\left(\left\|\boldsymbol{e}_{u_{t}}\right\|_{\Omega}^{2}+\left\|\boldsymbol{e}_{\boldsymbol{p}}\right\|_{\Omega}^{2}\right) d t \leq C h^{2 k+2},
\end{gathered}
$$

where $C$ depends on $\|u\|_{L^{\infty}\left((0, T) ; H^{k+4}(\Omega)\right)},\left\|u_{t}\right\|_{L^{\infty}\left((0, T) ; H^{k+4}(\Omega)\right)},\|\boldsymbol{r}\|_{\infty},\left\|\boldsymbol{r}_{t}\right\|_{\infty}$, $\|H\|_{\infty},\left\|H_{t}\right\|_{\infty},\|\boldsymbol{p}\|_{\infty},\left\|u_{t}\right\|_{\infty}, T$.

Recalling Lemma 5.4, Lemma 5.5, Lemma 5.7, Lemma 5.8, we obtain estimates

$$
\begin{array}{r}
\max _{t}\left(\left\|\boldsymbol{e}_{\boldsymbol{q}}\right\|_{\Omega}^{2}+\left\|\boldsymbol{e}_{W}\right\|_{\Omega}^{2}+\left\|\boldsymbol{e}_{\boldsymbol{v}}\right\|_{\Omega}^{2}\right) \leq C h^{2 k+2}, \\
\int_{0}^{T}\left\|\boldsymbol{e}_{\boldsymbol{s}}\right\|_{\Omega}^{2} d t \leq C h^{2 k+2} .
\end{array}
$$

Recalling Lemma 5.2 and Lemma 5.1, we can also get the following estimates

$$
\left\|u-u_{h}\right\|_{\Omega} \leq C h^{k+1}
$$


To complete the proof, let us verify the a priori assumptions (4.4)-(4.5). For $k \geq 1$ and $d \leq 3$, we can consider $h$ small enough so that $C h^{k+1}<\frac{1}{2} h^{\frac{7}{4}}$, where $C$ is the constant determined by the final time $T$. Then, if $t^{*}=\sup \left\{t:\left\|\boldsymbol{r}(s)-\boldsymbol{r}_{h}(s)\right\| \leq\right.$ $\left.h^{\frac{7}{4}}, \quad\left\|H(s)-H_{h}(s)\right\| \leq h^{\frac{7}{4}}, s \in[0, t)\right\}$, we would have $\left\|\boldsymbol{r}\left(t^{*}\right)-\boldsymbol{r}_{h}\left(t^{*}\right)\right\|=h^{\frac{7}{4}}$, $\left\|H\left(t^{*}\right)-H_{h}\left(t^{*}\right)\right\|=h^{\frac{7}{4}}$ by continuity if $t^{*}$ is finite. On the other hand, our proof implies that (4.4) and (4.5) holds for $t \leq t^{*}$, in particular

$$
\left\|\boldsymbol{r}\left(t^{*}\right)-\boldsymbol{r}_{h}\left(t^{*}\right)\right\| \leq C h^{k+\frac{1}{2}}<\frac{1}{2} h^{\frac{7}{4}}, \quad\left\|H\left(t^{*}\right)-H_{h}\left(t^{*}\right)\right\| \leq C h^{k+\frac{1}{2}}<\frac{1}{2} h^{\frac{7}{4}} .
$$

This is a contradiction if $t^{*}<T$. Hence $t^{*} \geq T$ and our a priori assumptions (4.4) and (4.5) are justified when $d \leq 3$.

\section{Concluding remarks}

In this paper, we have presented the optimal error analysis for the LDG method of the Willmore flow of graphs on Cartesian meshes. The analysis is made for the fully nonlinear case and the results are valid for all space dimension $d \leq 3$ and polynomial degree $k \geq 1$. And our results for $\left\|u-u_{h}\right\|_{\Omega}$ is just true for one dimension. Another important issue not addressed in this paper is $L^{2}$ a priori error estimates on triangular meshes. If we follow the same proof technique in this paper for triangular meshes, we could easily lose half an order or even one order in accuracy, because of a lack of control for certain jump terms at cell boundaries and difficulty from the nonlinear terms. Such error estimates are left for future work.

\section{Appendix A. Appendix: Proof of several Lemmas}

A.1. Proof of Lemma 4.1. We obverse that the second inequality of the (4.1) is a consequence of the first inequality. Recalling the definition of $\gamma$ and $\gamma_{h}$, we have

$$
\gamma-\gamma_{h}=\left(-\frac{r}{Q}+\frac{\boldsymbol{r}_{h}}{Q_{h}}, \frac{1}{Q}-\frac{1}{Q_{h}}\right)^{T}
$$

Obviously,

$$
\left|\gamma-\gamma_{h}\right|^{2}=\left|\frac{\boldsymbol{r}}{Q}-\frac{\boldsymbol{r}_{h}}{Q_{h}}\right|^{2}+\left(\frac{1}{Q}-\frac{1}{Q_{h}}\right)^{2}
$$

We get

$$
\left|\frac{1}{Q}-\frac{1}{Q_{h}}\right| \leq\left|\gamma-\gamma_{h}\right|, \quad\left|\frac{\boldsymbol{r}}{Q}-\frac{\boldsymbol{r}_{h}}{Q_{h}}\right| \leq\left|\gamma-\gamma_{h}\right| .
$$

To prove (4.2), let us introduce the notation $z=\frac{r}{Q}, z_{h}=\frac{r_{h}}{Q_{h}}$, thus

$$
\begin{aligned}
\left|\frac{\boldsymbol{r} \otimes \boldsymbol{r}}{Q}-\frac{\boldsymbol{r}_{h} \otimes \boldsymbol{r}_{h}}{Q_{h}}\right| & =\left|\boldsymbol{z} \otimes \boldsymbol{z} Q-\boldsymbol{z}_{h} \otimes \boldsymbol{z}_{h} Q_{h}\right| \\
& =\left|\left(\boldsymbol{z}-\boldsymbol{z}_{h}\right) \otimes \boldsymbol{z} Q+\boldsymbol{z}_{h} \otimes \boldsymbol{z}\left(Q-Q_{h}\right)+\boldsymbol{z}_{h} \otimes\left(\boldsymbol{z}-\boldsymbol{z}_{h}\right) Q_{h}\right| .
\end{aligned}
$$

Therefore, the triangle inequality and the fact that $\left|\boldsymbol{z}-\boldsymbol{z}_{h}\right| \leq\left|\boldsymbol{\gamma}-\boldsymbol{\gamma}_{h}\right|$ yield (4.2). It remains only to demonstrate (4.3). By the definition of $\gamma$ and $\gamma_{h}$,

$$
(-\boldsymbol{r}, 1)^{T}=\gamma Q, \quad\left(-\boldsymbol{r}_{h}, 1\right)^{T}=\gamma_{h} Q_{h},
$$

We have

$$
\left(\boldsymbol{r}-\boldsymbol{r}_{h}, 0\right)^{T}=\gamma_{h} Q_{h}-\gamma Q
$$

In view of $|\gamma|=\left|\gamma_{h}\right|=1$

$$
\left|\boldsymbol{r}-\boldsymbol{r}_{h}\right|^{2}=\left(Q-Q_{h}\right)^{2}+\left|\boldsymbol{\gamma}-\boldsymbol{\gamma}_{h}\right|^{2} Q Q_{h} .
$$


Clearly, $Q \geq 1$ and $Q_{h} \geq 1$, we have

$$
\left|\gamma-\gamma_{h}\right|^{2} \leq\left|\gamma-\gamma_{h}\right|^{2} Q Q_{h} \leq\left|\boldsymbol{r}-\boldsymbol{r}_{h}\right|^{2} .
$$

Consequently, we obtain (4.3).

A.2. Proof of Lemma 4.2. For any vector $\boldsymbol{p}$ and $\boldsymbol{q}$ we define $\gamma_{1}$ and $\gamma_{2}$ as follows

We denote

$$
\gamma_{1}=\frac{(-\boldsymbol{p}, 1)^{T}}{R_{1}}, \quad \gamma_{2}=\frac{(-\boldsymbol{q}, 1)^{T}}{R_{2}}
$$

$$
R_{1}=\sqrt{1+|\boldsymbol{p}|^{2}}, \quad R_{2}=\sqrt{1+|\boldsymbol{q}|^{2}},
$$

then

$$
R_{1}^{2}=1+|\boldsymbol{p}|^{2}, \quad R_{2}^{2}=1+|\boldsymbol{q}|^{2} .
$$

According to the definition of $\boldsymbol{E}(\boldsymbol{p})$, we have

And

$$
|\boldsymbol{E}(\boldsymbol{p})|=\left|\frac{1}{R_{1}}\left(\boldsymbol{I}-\frac{\boldsymbol{p} \otimes \boldsymbol{p}}{R_{1}^{2}}\right)\right| \leq\left|\frac{1}{R_{1}}\right|\left(1+\frac{|\boldsymbol{p}|^{2}}{R_{1}^{2}}\right) \leq 2 .
$$

$$
\begin{aligned}
\boldsymbol{E}(\boldsymbol{p}) \boldsymbol{q} \cdot \boldsymbol{q} & =\frac{\boldsymbol{q} \cdot \boldsymbol{q}}{R_{1}}-\frac{|\boldsymbol{p} \cdot \boldsymbol{q}|^{2}}{R_{1}^{3}}=\frac{1}{R_{1}^{3}}\left(|\boldsymbol{q}|^{2} R_{1}^{2}-|\boldsymbol{p} \cdot \boldsymbol{q}|^{2}\right) \\
& \geq \frac{1}{R_{1}^{3}}\left(|\boldsymbol{q}|^{2} R_{1}^{2}-|\boldsymbol{p}|^{2}|\boldsymbol{q}|^{2}\right) \\
& =\frac{|\boldsymbol{q}|^{2}}{R_{1}^{3}}\left(R_{1}^{2}-|\boldsymbol{p}|^{2}\right)=\frac{|\boldsymbol{q}|^{2}}{R_{1}^{3}}=\frac{|\boldsymbol{q}|^{2}}{{\sqrt{1+|\boldsymbol{p}|^{2}}}^{3}} .
\end{aligned}
$$

Now we finish the proof of (4.11).

We follow the proof in A.1, we have

$$
\left|\frac{1}{R_{1}}-\frac{1}{R_{2}}\right| \leq\left|\gamma_{1}-\gamma_{2}\right|, \quad\left|\frac{\boldsymbol{p}}{R_{1}}-\frac{\boldsymbol{q}}{R_{2}}\right| \leq\left|\gamma_{1}-\gamma_{2}\right| .
$$

Let us introduce the notation $\boldsymbol{z}_{1}=\frac{p}{R_{1}}, \boldsymbol{z}_{2}=\frac{q}{R_{2}}$, so

$$
\left|z_{1}-z_{2}\right|=\left|\frac{\boldsymbol{p}}{R_{1}}-\frac{\boldsymbol{q}}{R_{2}}\right| \leq\left|\gamma_{1}-\gamma_{2}\right|
$$

thus we can obtain

$$
\begin{aligned}
\left|\frac{\boldsymbol{p} \otimes \boldsymbol{p}}{R_{1}^{3}}-\frac{\boldsymbol{q} \otimes \boldsymbol{q}}{R_{2}^{3}}\right| & =\left|\frac{\boldsymbol{z}_{1} \otimes \boldsymbol{z}_{1}}{R_{1}}-\frac{\boldsymbol{z}_{2} \otimes \boldsymbol{z}_{2}}{R_{2}}\right| \\
& =\left|\left(\boldsymbol{z}_{1}-\boldsymbol{z}_{2}\right) \otimes \boldsymbol{z}_{1} \frac{1}{R_{1}}\right|+\left|\boldsymbol{z}_{2} \otimes \boldsymbol{z}_{1}\left(\frac{1}{R_{1}}-\frac{1}{R_{2}}\right)\right|+\left|\boldsymbol{z}_{2} \otimes\left(\boldsymbol{z}_{1}-\boldsymbol{z}_{2}\right) \frac{1}{R_{2}}\right| \\
& \leq 3\left|\gamma_{1}-\gamma_{2}\right| .
\end{aligned}
$$

By the definition of $\gamma_{1}$ and $\gamma_{2}$,

$$
(-\boldsymbol{p}, 1)^{T}=\gamma_{1} R_{1}, \quad(-\boldsymbol{q}, 1)^{T}=\gamma_{2} R_{2},
$$

we have

$$
(\boldsymbol{p}-\boldsymbol{q}, 0)^{T}=\gamma_{2} R_{2}-\gamma_{1} R_{1} .
$$

In view of $\left|\gamma_{1}\right|=\left|\gamma_{2}\right|=1$,

$$
|\boldsymbol{p}-\boldsymbol{q}|^{2}=\left(R_{1}-R_{2}\right)^{2}+\left|\gamma_{1}-\gamma_{2}\right|^{2} R_{1} R_{2} .
$$

Clearly, $R_{1} \geq 1$ and $R_{2} \geq 1$, we have

$$
\left|\gamma_{1}-\gamma_{2}\right|^{2} \leq\left|\gamma_{1}-\gamma_{2}\right|^{2} R_{1} R_{2} \leq|\boldsymbol{p}-\boldsymbol{q}|^{2} .
$$


So we finally get

$$
\begin{aligned}
|\boldsymbol{E}(\boldsymbol{p})-\boldsymbol{E}(\boldsymbol{q})| & =\left|\boldsymbol{I}\left(\frac{1}{R_{1}}-\frac{1}{R_{2}}\right)-\left(\frac{\boldsymbol{p} \otimes \boldsymbol{p}}{R_{1}^{3}}-\frac{\boldsymbol{q} \otimes \boldsymbol{q}}{R_{2}^{3}}\right)\right| \\
& \leq\left|\frac{1}{R_{1}}-\frac{1}{R_{2}}\right|+\left|\frac{\boldsymbol{p} \otimes \boldsymbol{p}}{R_{1}^{3}}-\frac{\boldsymbol{q} \otimes \boldsymbol{q}}{R_{2}^{3}}\right| \\
& \leq 4\left|\gamma_{1}-\gamma_{2}\right| \leq 4|\boldsymbol{p}-\boldsymbol{q}| .
\end{aligned}
$$

A.3. Proof of Lemma 5.1. For the given the initial function $u_{0}(x)$, we choose

$$
\boldsymbol{q}_{h}(x, 0)=\Pi^{+} \boldsymbol{q}(x, 0), \boldsymbol{q}(x, 0)=\frac{\nabla u_{0}(x)}{\sqrt{1+\left|\nabla u_{0}(x)\right|^{2}}} .
$$

The initial data $u_{h}(x, 0)$ is the solution of the following equations

$$
\begin{aligned}
& \int_{K} H_{h} \vartheta d K+\int_{K} \boldsymbol{q}_{h} \cdot \nabla \vartheta d K-\int_{\partial K} \widehat{\boldsymbol{q}_{h} \cdot \boldsymbol{\nu}} \vartheta d s=0, \\
& \int_{K} \boldsymbol{q}_{h} \cdot \boldsymbol{\rho} d K-\int_{K} \frac{\boldsymbol{r}_{h}}{Q_{h}} \cdot \boldsymbol{\rho} d K=0, \\
& \int_{K} \boldsymbol{r}_{h} \cdot \boldsymbol{\zeta} d K+\int_{K} u_{h} \nabla \cdot \boldsymbol{\zeta} d K-\int_{\partial K} \widehat{u_{h}} \boldsymbol{\nu} \cdot \boldsymbol{\zeta} d s=0,
\end{aligned}
$$

and also satisfies

$$
\int_{\Omega} u(x, 0) d \Omega=\int_{\Omega} u_{h}(x, 0) d \Omega
$$

where $\forall \vartheta \in V_{h}$ and $\forall \boldsymbol{\rho}, \boldsymbol{\zeta} \in \Sigma_{h}$.

For given $\boldsymbol{q}_{h}$, we can easily see that $\boldsymbol{r}_{h}$ is well-defined. Now we use $\boldsymbol{r}_{h}$ to find a well-defined $u_{h}$. We consider the elliptic linear problem

$$
\begin{aligned}
-\boldsymbol{\zeta}^{*} & =\nabla \xi^{*}, \quad \text { in } \quad \Omega \\
\eta^{*} & =\nabla \cdot \boldsymbol{\zeta}^{*}, \quad \text { in } \Omega
\end{aligned}
$$

with the periodic boundary conditions. To make the problem well-defined, we should assume that the average of $\varphi^{*}$ on $\Omega$ is a given constant and that of $\eta^{*}$ is zero. We have the elliptic regularity result

$$
\left\|\boldsymbol{\zeta}^{*}\right\|_{H^{1}\left(\Omega_{h}\right)}+\left\|\xi^{*}\right\|_{H^{2}\left(\Omega_{h}\right)} \leq C\left\|\eta^{*}\right\|_{L^{2}\left(\Omega_{h}\right)} .
$$

The existence is obvious. We know very well that if $u_{h}$ satisfies (A.4) then $u_{h}+c$ also satisfies (A.4). Here $c$ is any constant. If there are two solutions $u_{h 1}$ and $u_{h 2}$ both satisfying (A.4) and the assumption (A.5)

$$
\int_{\Omega} u(x, 0) d \Omega=\int_{\Omega} u_{h 1}(x, 0) d \Omega=\int_{\Omega} u_{h 2}(x, 0) d \Omega
$$

then we can easily get

$$
\begin{aligned}
& \int_{K}\left(u_{h 1}-u_{h 2}\right) \nabla \cdot \boldsymbol{\zeta} d K-\int_{\partial K}\left(\widehat{u_{h 1}}-\widehat{u_{h 2}}\right) \boldsymbol{\nu} \cdot \boldsymbol{\zeta} d s=0, \\
& \int_{\Omega}\left(u_{h 1}(x, 0)-u_{h 2}(x, 0)\right) d \Omega=0 .
\end{aligned}
$$

Taking $\eta^{*}=u_{h 1}(x, 0)-u_{h 2}(x, 0)$ in the corresponding elliptic linear equation we get

$$
\begin{aligned}
& \left(u_{h 1}-u_{h 2}, u_{h 1}-u_{h 2}\right)_{K} \\
= & \left(u_{h 1}-u_{h 2}, \nabla \cdot \boldsymbol{\zeta}^{*}\right)_{K} \\
= & \left(u_{h 1}-u_{h 2}, \nabla \cdot\left(\boldsymbol{\zeta}^{*}-\Pi \boldsymbol{\zeta}^{*}\right)\right)_{K}+\left(u_{h 1}-u_{h 2}, \nabla \cdot \Pi \boldsymbol{\zeta}^{*}\right)_{K}
\end{aligned}
$$




$$
\begin{aligned}
= & \left(u_{h 1}-u_{h 2}, \nabla \cdot\left(\boldsymbol{\zeta}^{*}-\Pi \boldsymbol{\zeta}^{*}\right)\right)_{K}-<\widehat{u_{h 1}}-\widehat{u_{h 2}}, \boldsymbol{\nu} \cdot\left(\boldsymbol{\zeta}^{*}-\Pi \boldsymbol{\zeta}^{*}\right)>_{\partial K}+<\widehat{u_{h 1}}-\widehat{u_{h 2}}, \boldsymbol{\nu} \cdot \boldsymbol{\zeta}^{*}>_{\partial K} \\
= & -\left(\nabla\left(u_{h 1}-u_{h 2}\right), \boldsymbol{\zeta}^{*}-\Pi \boldsymbol{\zeta}^{*}\right)_{K}+<u_{h 1}-u_{h 2}, \boldsymbol{\nu} \cdot\left(\boldsymbol{\zeta}^{*}-\Pi \boldsymbol{\zeta}^{*}\right)>_{\partial K} \\
& -<\widehat{u_{h 1}}-\widehat{u_{h 2}}, \boldsymbol{\nu} \cdot\left(\boldsymbol{\zeta}^{*}-\Pi \boldsymbol{\zeta}^{*}\right)>_{\partial K}+<\widehat{u_{h 1}}-\widehat{u_{h 2}}, \boldsymbol{\nu} \cdot \boldsymbol{\zeta}^{*}>_{\partial K}
\end{aligned}
$$

Recalling that $\widehat{u_{h}}=u_{h}^{-}$, we take $\Pi \zeta^{*}=\Pi^{+} \zeta^{*}$ and sum over $K$. By the continuity of $\boldsymbol{\zeta}^{*}$ and the definition of the projection $\Pi^{+}$we obtain

$$
\left(u_{h 1}-u_{h 2}, u_{h 1}-u_{h 2}\right)=0 .
$$

Then we get $u_{h 1}=u_{h 2}$. Finally we have proved that $u_{h}$ is well-defined.

In the following, we will give the proof of the error estimate in Lemma 5.1. We have the error equations

$$
\begin{aligned}
& \int_{K}\left(H-H_{h}\right) \vartheta d K+\int_{K}\left(\boldsymbol{q}-\boldsymbol{q}_{h}\right) \cdot \nabla \vartheta d K-\int_{\partial K}\left(\widehat{\boldsymbol{q - q _ { h }}}\right) \cdot \boldsymbol{\nu} \vartheta d s=0, \\
& \int_{K}\left(\boldsymbol{q}-\boldsymbol{q}_{h}\right) \cdot \boldsymbol{\rho} d K-\int_{K}\left(\frac{\boldsymbol{r}}{Q}-\frac{\boldsymbol{r}_{h}}{Q_{h}}\right) \cdot \boldsymbol{\rho} d K=0, \\
& \int_{K}\left(\boldsymbol{r}-\boldsymbol{r}_{h}\right) \cdot \boldsymbol{\zeta} d K+\int_{K}\left(u-u_{h}\right) \nabla \cdot \boldsymbol{\zeta} d K-\int_{\partial K}\left(\widehat{u-u_{h}}\right) \boldsymbol{\nu} \cdot \boldsymbol{\zeta} d s=0 .
\end{aligned}
$$

From the property of the special projection we have known that

$$
\left\|\boldsymbol{q}(x, 0)-\boldsymbol{q}_{h}(x, 0)\right\|_{\Omega}=\left\|\boldsymbol{q}(x, 0)-\Pi^{+} \boldsymbol{q}(x, 0)\right\|_{\Omega} \leq C h^{k+1}
$$

and (A.6) becomes

$$
\int_{K}\left(H-H_{h}\right) \vartheta d K+\int_{K}\left(\boldsymbol{q}-\Pi^{+} \boldsymbol{q}\right) \cdot \nabla \vartheta d K-\int_{\partial K}\left(\widehat{\boldsymbol{q}} \widehat{-\Pi^{+}} \boldsymbol{q}\right) \cdot \boldsymbol{\nu} \vartheta d s=0,
$$

Taking $\vartheta=P^{-} H-H_{h}$ and summing over $K$ we can get the estimate

$$
\left\|H(x, 0)-H_{h}(x, 0)\right\|_{\Omega} \leq C h^{k+1}
$$

according to the special projection and fluxes we choose. Now we use (A.7) to estimate $\left\|\boldsymbol{r}-\boldsymbol{r}_{h}\right\|_{\Omega}$ by taking $\boldsymbol{\rho}=\left(\boldsymbol{r}_{h}-\Pi \boldsymbol{r}\right)$

$$
\begin{aligned}
0= & \int_{K}\left(\boldsymbol{q}-\boldsymbol{q}_{h}\right) \cdot\left(\boldsymbol{r}_{h}-\Pi \boldsymbol{r}\right) d K-\int_{K}\left(\frac{\boldsymbol{r}}{Q}-\frac{\boldsymbol{r}_{h}}{Q_{h}}\right) \cdot\left(\boldsymbol{r}_{h}-\Pi \boldsymbol{r}\right) d K \\
= & \int_{K}\left(\boldsymbol{q}-\boldsymbol{q}_{h}\right) \cdot\left(\boldsymbol{r}_{h}-\Pi \boldsymbol{r}\right) d K \\
& -\int_{K}\left(\frac{\boldsymbol{r}}{Q}-\frac{\Pi \boldsymbol{r}}{\widetilde{Q_{h}}}\right) \cdot\left(\boldsymbol{r}_{h}-\Pi \boldsymbol{r}\right) d K-\int_{K}\left(\frac{\Pi \boldsymbol{r}}{\widetilde{Q_{h}}}-\frac{\boldsymbol{r}_{h}}{Q_{h}}\right) \cdot\left(\boldsymbol{r}_{h}-\Pi \boldsymbol{r}\right) d K .
\end{aligned}
$$

Here we denote $\widetilde{Q_{h}}=\sqrt{1+|\Pi \boldsymbol{r}|^{2}}$. Recalling that

$$
\gamma=\frac{(-\boldsymbol{r}, 1)^{T}}{Q}, \quad \gamma_{h}=\frac{\left(-\boldsymbol{r}_{h}, 1\right)^{T}}{Q_{h}}
$$

similarly we denote

$$
\widetilde{\gamma_{h}}=\frac{(-\Pi r, 1)^{T}}{\widetilde{Q_{h}}}
$$

then we easily get

$$
\left(\boldsymbol{r}_{h}-\Pi \boldsymbol{r}, 0\right)=\widetilde{\gamma_{h}} \widetilde{Q_{h}}-\gamma_{h} Q_{h}
$$

and also

$$
\left(\frac{\Pi \boldsymbol{r}}{\widetilde{Q_{h}}}-\frac{\boldsymbol{r}_{h}}{Q_{h}}\right) \cdot\left(\boldsymbol{r}_{h}-\Pi \boldsymbol{r}\right)
$$




$$
\begin{aligned}
& =\frac{\Pi \boldsymbol{r}}{\widetilde{Q_{h}}} \cdot\left(\boldsymbol{r}_{h}-\Pi \boldsymbol{r}\right)-\frac{\boldsymbol{r}_{h}}{Q_{h}} \cdot\left(\boldsymbol{r}_{h}-\Pi \boldsymbol{r}\right) \\
& =-\left(-\frac{\Pi \boldsymbol{r}}{\widetilde{Q_{h}}}, \frac{1}{\widetilde{Q_{h}}}\right) \cdot\left(\boldsymbol{r}_{h}-\Pi \boldsymbol{r}, 0\right)+\left(-\frac{\boldsymbol{r}_{h}}{Q_{h}}, \frac{1}{Q_{h}}\right) \cdot\left(\boldsymbol{r}_{h}-\Pi \boldsymbol{r}, 0\right) \\
& =-\widetilde{\gamma_{h}} \cdot\left(\widetilde{\gamma_{h}} \widetilde{Q_{h}}-\gamma_{h} Q_{h}\right)+\gamma_{h} \cdot\left(\widetilde{\gamma_{h}} \widetilde{Q_{h}}-\gamma_{h} Q_{h}\right) \\
& =-\left(\widetilde{\gamma_{h}}-\gamma_{h}\right) \cdot\left(\widetilde{\gamma_{h}} \widetilde{Q_{h}}-\gamma_{h} Q_{h}\right) \\
& =-\left(\widetilde{Q_{h}}+Q_{h}\right)\left(1-\widetilde{\gamma_{h}} \cdot \gamma_{h}\right) \\
& =-\frac{1}{2}\left|\widetilde{\gamma_{h}}-\gamma_{h}\right|^{2}\left(\widetilde{Q_{h}}+Q_{h}\right)
\end{aligned}
$$

Consider

$$
\Pi \boldsymbol{r}-\boldsymbol{r}_{h}=\widetilde{Q_{h}}\left(\frac{\Pi \boldsymbol{r}}{\widetilde{Q_{h}}}-\frac{\boldsymbol{r}_{h}}{Q_{h}}\right)-\widetilde{Q_{h}}\left(\frac{1}{\widetilde{Q_{h}}}-\frac{1}{Q_{h}}\right) \boldsymbol{r}_{h} .
$$

Using (4.1) and (4.2), we have

$$
\left|\Pi \boldsymbol{r}-\boldsymbol{r}_{h}\right| \leq \widetilde{Q_{h}}\left|\widetilde{\gamma_{h}}-\gamma_{h}\right|+\widetilde{Q_{h}}\left|\widetilde{\gamma_{h}}-\gamma_{h}\right|\left|\boldsymbol{r}_{h}\right| .
$$

Thanks to a priori assumption, we have

$$
\left|\Pi \boldsymbol{r}-\boldsymbol{r}_{h}\right|^{2} \leq C\left\|\widetilde{Q_{h}}\right\|_{\infty}^{2}\left|\widetilde{\gamma_{h}}-\gamma_{h}\right|^{2}\left(1+\left|\boldsymbol{r}_{h}^{2}\right|\right)=C\left\|\widetilde{Q_{h}}\right\|_{\infty}^{2}\left|\widetilde{\gamma_{h}}-\gamma_{h}\right|^{2} Q_{h}^{2} \leq C\left|\widetilde{\gamma_{h}}-\gamma_{h}\right|^{2},
$$

where $C$ depends on $\|Q\|_{\infty}$ and constant $R$ defined in (4.9). Then we have

$$
\frac{1}{2}\left|\widetilde{\gamma_{h}}-\gamma_{h}\right|^{2}\left(\widetilde{Q_{h}}+Q_{h}\right) \geq\left|\widetilde{\gamma_{h}}-\gamma_{h}\right|^{2} \geq C\left|\Pi \boldsymbol{r}-\boldsymbol{r}_{h}\right|^{2}
$$

Now we have

$$
\begin{aligned}
0= & \int_{K}\left(\boldsymbol{q}-\boldsymbol{q}_{h}\right) \cdot\left(\boldsymbol{r}_{h}-\Pi \boldsymbol{r}\right) d K-\int_{K}\left(\frac{\boldsymbol{r}}{Q}-\frac{\boldsymbol{r}_{h}}{Q_{h}}\right) \cdot\left(\boldsymbol{r}_{h}-\Pi \boldsymbol{r}\right) d K \\
= & \int_{K}\left(\boldsymbol{q}-\boldsymbol{q}_{h}\right) \cdot\left(\boldsymbol{r}_{h}-\Pi \boldsymbol{r}\right) d K \\
& -\int_{K}\left(\frac{\boldsymbol{r}}{Q}-\frac{\Pi \boldsymbol{r}}{\widetilde{Q_{h}}}\right) \cdot\left(\boldsymbol{r}_{h}-\Pi \boldsymbol{r}\right) d K+\int_{K} \frac{1}{2}\left|\widetilde{\gamma_{h}}-\gamma_{h}\right|^{2}\left(\widetilde{Q_{h}}+Q_{h}\right) d K
\end{aligned}
$$

Then we have

$$
\begin{aligned}
C \int_{K}\left|\Pi \boldsymbol{r}-\boldsymbol{r}_{h}\right|^{2} d K & \leq \int_{K} \frac{1}{2}\left|\widetilde{\gamma_{h}}-\gamma_{h}\right|^{2}\left(\widetilde{Q_{h}}+Q_{h}\right) d K \\
& =-\int_{K}\left(\boldsymbol{q}-\boldsymbol{q}_{h}\right) \cdot\left(\boldsymbol{r}_{h}-\Pi \boldsymbol{r}\right) d K+\int_{K}\left(\frac{\boldsymbol{r}}{Q}-\frac{\Pi \boldsymbol{r}}{\widetilde{Q_{h}}}\right) \cdot\left(\boldsymbol{r}_{h}-\Pi \boldsymbol{r}\right) d K
\end{aligned}
$$

Using Cauchy-Schwarz inequality we get the following estimate

$$
\left\|\Pi \boldsymbol{r}(x, 0)-\boldsymbol{r}_{h}(x, 0)\right\|_{\Omega} \leq C h^{k+1}, \quad\left\|\boldsymbol{r}(x, 0)-\boldsymbol{r}_{h}(x, 0)\right\|_{\Omega} \leq C h^{k+1} .
$$

Now we estimate $\left\|u-u_{h}\right\|_{\Omega}$. We use the same technique above by taking $\eta^{*}=u-u_{h}$

$$
\begin{aligned}
\left(u-u_{h}, u-u_{h}\right)_{K}=\left(u-u_{h}, \nabla \cdot \boldsymbol{\zeta}^{*}\right)_{K} \\
=\left(u-u_{h}, \nabla \cdot\left(\boldsymbol{\zeta}^{*}-\Pi \boldsymbol{\zeta}^{*}\right)\right)_{K}+\left(u-u_{h}, \nabla \cdot \Pi \boldsymbol{\zeta}^{*}\right)_{K} \\
=\left(u-u_{h}, \nabla \cdot\left(\boldsymbol{\zeta}^{*}-\Pi \boldsymbol{\zeta}^{*}\right)\right)_{K}-<u-\widehat{u_{h}}, \boldsymbol{\nu} \cdot\left(\boldsymbol{\zeta}^{*}-\Pi \boldsymbol{\zeta}^{*}\right)>_{\partial K}+<u-\widehat{u_{h}}, \boldsymbol{\nu} \cdot \boldsymbol{\zeta}^{*}>_{\partial K} \\
\quad-\left(\boldsymbol{r}-\boldsymbol{r}_{h}, \Pi \boldsymbol{\zeta}^{*}-\boldsymbol{\zeta}^{*}\right)_{K}-\left(\boldsymbol{r}-\boldsymbol{r}_{h}, \boldsymbol{\zeta}^{*}\right)_{K} \\
=-\left(\nabla\left(u-u_{h}\right), \boldsymbol{\zeta}^{*}-\Pi \boldsymbol{\zeta}^{*}\right)_{K}+<u-u_{h}, \boldsymbol{\nu} \cdot\left(\boldsymbol{\zeta}^{*}-\Pi \boldsymbol{\zeta}^{*}\right)>_{\partial K} \\
\quad-<u-\widehat{u_{h}}, \boldsymbol{\nu} \cdot\left(\boldsymbol{\zeta}^{*}-\Pi \boldsymbol{\zeta}^{*}\right)>_{\partial K}+<u-\widehat{u_{h}}, \boldsymbol{\nu} \cdot \boldsymbol{\zeta}^{*}>_{\partial K}
\end{aligned}
$$




$$
\begin{aligned}
& -\left(\boldsymbol{r}-\boldsymbol{r}_{h}, \Pi \boldsymbol{\zeta}^{*}-\boldsymbol{\zeta}^{*}\right)_{K}-\left(\boldsymbol{r}-\boldsymbol{r}_{h}, \boldsymbol{\zeta}^{*}\right)_{K} \\
= & -\left(\nabla\left(u-P u+P u-u_{h}\right), \boldsymbol{\zeta}^{*}-\Pi \boldsymbol{\zeta}^{*}\right)_{K}+<\widehat{u_{h}}-u_{h}, \boldsymbol{\nu} \cdot\left(\boldsymbol{\zeta}^{*}-\Pi \boldsymbol{\zeta}^{*}\right)>_{\partial K} \\
& +<u-\widehat{u_{h}}, \boldsymbol{\nu} \cdot \boldsymbol{\zeta}^{*}>_{\partial K}-\left(\boldsymbol{r}-\boldsymbol{r}_{h}, \Pi \boldsymbol{\zeta}^{*}-\boldsymbol{\zeta}^{*}\right)_{K}-\left(\boldsymbol{r}-\boldsymbol{r}_{h}, \boldsymbol{\zeta}^{*}\right)_{K} .
\end{aligned}
$$

Recalling that $\widehat{u_{h}}=u_{h}^{-}$we take $\Pi \boldsymbol{\zeta}^{*}=\Pi^{+} \boldsymbol{\zeta}^{*}$ and sum over $K$. By the continuity of $\zeta^{*}$ and the definition of the projection $\Pi^{+}$we obtain

$$
\begin{aligned}
& \left(u-u_{h}, u-u_{h}\right) \\
& =-\sum_{K}\left(\nabla(u-P u), \boldsymbol{\zeta}^{*}-\Pi \boldsymbol{\zeta}^{*}\right)_{K}-\sum_{K}\left(\boldsymbol{r}-\boldsymbol{r}_{h}, \Pi \boldsymbol{\zeta}^{*}-\boldsymbol{\zeta}^{*}\right)_{K}-\sum_{K}\left(\boldsymbol{r}-\boldsymbol{r}_{h}, \boldsymbol{\zeta}^{*}\right)_{K} \\
& \leq C h^{k+1}\left\|\boldsymbol{\zeta}^{*}\right\|_{H^{1}\left(\Omega_{h}\right)}+C h^{k+2}\left\|\boldsymbol{\zeta}^{*}\right\|_{H^{1}\left(\Omega_{h}\right)}+C h^{k+1}\left\|\boldsymbol{\zeta}^{*}\right\|_{L^{2}\left(\Omega_{h}\right)} \\
& \leq C h^{k+1}\left\|\boldsymbol{\zeta}^{*}\right\|_{H^{1}\left(\Omega_{h}\right)} \\
& \leq C h^{k+1}\left\|u-u_{h}\right\|_{L^{2}\left(\Omega_{h}\right)} .
\end{aligned}
$$

Finally we got the estimate for the $\left\|u(x, 0)-u_{h}(x, 0)\right\|_{\Omega} \leq C h^{k+1}$.

A.4. Proof of Lemma 5.6. We consider the (3.4a)-(3.4d) to get the error equations

$$
\begin{aligned}
& \int_{K}\left(\frac{u_{t}}{Q}-\frac{\left(u_{h}\right)_{t}}{Q_{h}}\right) \varphi d K-\int_{K}\left(\left(\boldsymbol{s}-\boldsymbol{s}_{h}\right)-\left(\boldsymbol{v}-\boldsymbol{v}_{h}\right)\right) \cdot \nabla \varphi d K \\
& \left.+\int_{\partial K} \varphi\left((\boldsymbol{s}-\boldsymbol{v}) \widehat{-\left(\boldsymbol{s}_{h}\right.}-\boldsymbol{v}_{h}\right)\right) \cdot \boldsymbol{\nu} d s=0, \\
& \int_{K}\left(\boldsymbol{s}-\boldsymbol{s}_{h}\right) \cdot \boldsymbol{\phi} d K-\int_{K}\left(\boldsymbol{E}(\boldsymbol{r}) \boldsymbol{p}-\boldsymbol{E}\left(\boldsymbol{r}_{h}\right) \boldsymbol{p}_{h}\right) \cdot \boldsymbol{\phi} d K=0, \\
& \int_{K}\left(\boldsymbol{v}-\boldsymbol{v}_{h}\right) \cdot \boldsymbol{\psi} d K-\int_{K} \frac{1}{2}\left(\frac{H^{2}}{Q} \boldsymbol{r}-\frac{H_{h}^{2}}{Q_{h}} \boldsymbol{r}_{h}\right) \cdot \boldsymbol{\psi} d K=0, \\
& \left.\int_{K}\left(\boldsymbol{p}-\boldsymbol{p}_{h}\right) \cdot \boldsymbol{\eta} d K+\int_{K}\left(W-W_{h}\right) \nabla \cdot \boldsymbol{\eta} d K-\int_{\partial K}\left(\widehat{W-W_{h}}\right)\right) \boldsymbol{\eta} \cdot \boldsymbol{\nu} d s=0 .
\end{aligned}
$$

Choosing the test function $\varphi=P e_{W}, \phi=\Pi e_{p}, \psi=-\Pi e_{p}, \boldsymbol{\eta}=-\left(\Pi e_{s}-\Pi e_{v}\right)$, we obtain

$$
\int_{K}\left(\boldsymbol{E}(\boldsymbol{r}) \boldsymbol{p}-\boldsymbol{E}\left(\boldsymbol{r}_{h}\right) \boldsymbol{p}_{h}\right) \cdot \boldsymbol{e}_{\boldsymbol{p}} d K=(I)+(I I)+(I I I)+(I V),
$$

where

$$
\begin{aligned}
(I) & =\int_{K}\left(\boldsymbol{E}(\boldsymbol{r}) \boldsymbol{p}-\boldsymbol{E}\left(\boldsymbol{r}_{h}\right) \boldsymbol{p}_{h}\right) \cdot(\boldsymbol{p}-\Pi \boldsymbol{p}) d K, \\
(I I) & =\int_{K}\left(\frac{u_{t}}{Q}-\frac{\left(u_{h}\right)_{t}}{Q_{h}}\right) P \boldsymbol{e}_{W} d K, \\
(I I I) & =\int_{K} \frac{1}{2}\left(\frac{H^{2}}{Q} \boldsymbol{r}-\frac{H_{h}^{2}}{Q_{h}} \boldsymbol{r}_{h}\right) \cdot \Pi \boldsymbol{e}_{\boldsymbol{p}} d K, \\
(I V) & =-\int_{K}\left((\boldsymbol{s}-\Pi \boldsymbol{s})-\left(\boldsymbol{v}-\boldsymbol{v}_{h}\right)\right) \cdot \nabla P \boldsymbol{e}_{W} d K+\int_{\partial K}((\boldsymbol{s}-\Pi \widehat{\boldsymbol{s}})-(\boldsymbol{v}-\Pi \boldsymbol{v})) \cdot \boldsymbol{\nu} P \boldsymbol{e}_{W} d s \\
& +\int_{K}(\boldsymbol{s}-\Pi \boldsymbol{s}) \cdot \Pi \boldsymbol{e}_{\boldsymbol{p}} d K-\int_{K}(\boldsymbol{v}-\Pi \boldsymbol{v}) \cdot \Pi \boldsymbol{e}_{\boldsymbol{p}} d K-\int_{K}(\boldsymbol{p}-\Pi \boldsymbol{p}) \cdot\left(\Pi \boldsymbol{e}_{\boldsymbol{s}}-\Pi \boldsymbol{e}_{\boldsymbol{v}}\right) d K \\
& -\int_{K}(W-P W) \nabla \cdot\left(\Pi \boldsymbol{e}_{\boldsymbol{s}}-\Pi \boldsymbol{e}_{\boldsymbol{v}}\right) d K+\int_{\partial K}(\widehat{W-P W})\left(\Pi \boldsymbol{e}_{\boldsymbol{s}}-\Pi \boldsymbol{e}_{\boldsymbol{v}}\right) \cdot \boldsymbol{\nu} d s .
\end{aligned}
$$

Now, we estimate $(I),(I I),(I I I),(I V)$, separately.

- Estimate $(I)$. 
Adding and subtracting $\boldsymbol{E}\left(\boldsymbol{r}_{h}\right) \boldsymbol{p} \cdot(\boldsymbol{p}-\Pi \boldsymbol{p})$, we have

$$
\begin{aligned}
(I) & =\int_{K}\left(\boldsymbol{E}(\boldsymbol{r}) \boldsymbol{p}-\boldsymbol{E}\left(\boldsymbol{r}_{h}\right) \boldsymbol{p}_{h}\right) \cdot(\boldsymbol{p}-\Pi \boldsymbol{p}) d K \\
& =\int_{K}\left(\boldsymbol{E}(\boldsymbol{r}) \boldsymbol{p}-\boldsymbol{E}\left(\boldsymbol{r}_{h}\right) \boldsymbol{p}\right) \cdot(\boldsymbol{p}-\Pi \boldsymbol{p}) d K+\int_{K}\left(\boldsymbol{E}\left(\boldsymbol{r}_{h}\right) \boldsymbol{e}_{\boldsymbol{p}}\right) \cdot(\boldsymbol{p}-\Pi \boldsymbol{p}) d K .
\end{aligned}
$$

Using Lemma 4.2 and Cauchy-Schwarz inequality, we derive

$$
\left|\sum_{K}\right| \leq \epsilon\left\|\boldsymbol{e}_{\boldsymbol{p}}\right\|_{\Omega}^{2}+C\left(h^{2 k+2}+N_{h}(t)\right)
$$

with C depending on $\epsilon$ and $\|\boldsymbol{p}\|_{H^{k+1}(\Omega)},\|\boldsymbol{p}\|_{\infty}$

- Estimate $(I I)$.

$$
\begin{aligned}
(I I) & =\int_{K}\left(\frac{u_{t}}{Q}-\frac{\left(u_{h}\right)_{t}}{Q_{h}}\right) P \boldsymbol{e}_{W} d K \\
& =\int_{K}\left(u_{t}\left(\frac{1}{Q}-\frac{1}{Q_{h}}\right)+\frac{\boldsymbol{e}_{u_{t}}}{Q_{h}}\right) P \boldsymbol{e}_{W} d K .
\end{aligned}
$$

Employing Cauchy-Schwarz inequality, we obtain

$$
\begin{aligned}
|(I I)| & \leq \int_{K}\left|u_{t}\right|\left|\frac{1}{Q}-\frac{1}{Q_{h}}\right|\left|P \boldsymbol{e}_{W}\right| d K+\int_{K} \frac{\left|\boldsymbol{e}_{u_{t}}\right|}{\sqrt{Q_{h}}} \frac{\left|P \boldsymbol{e}_{W}\right|}{\sqrt{Q_{h}}} d K \\
& \leq \frac{1}{2} \int_{K}\left|P \boldsymbol{e}_{W}\right|^{2} d K+\frac{1}{2}\left\|u_{t}\right\|_{\infty}^{2} \int_{K}\left(\frac{1}{Q}-\frac{1}{Q_{h}}\right)^{2} d K \\
& +\epsilon \int_{K} \frac{\boldsymbol{e}_{u_{t}}^{2}}{Q_{h}} d K+\frac{1}{4 \epsilon} \int_{K} \frac{\left|P \boldsymbol{e}_{W}\right|^{2}}{Q_{h}} d K .
\end{aligned}
$$

Recalling Lemma 5.7, we add all the elements $K$ to obtain

$$
\left|\sum_{K}(I I)\right| \leq \epsilon \sum_{K} \int_{K} \frac{e_{u_{t}}^{2}}{Q_{h}} d K+C\left(N_{h}(t)+h^{2 k+2}+\left\|\boldsymbol{e}_{H} \sqrt{Q_{h}}\right\|_{\Omega}^{2}\right),
$$

where $C$ depends on $\epsilon,\left\|u_{t}\right\|_{\infty},\|\boldsymbol{r}\|_{\infty},\|H\|_{\infty},\|W\|_{H^{k+1}(\Omega)}$.

- Estimate $(I I I)$.

$$
\begin{aligned}
(I I I) & =\int_{K} \frac{1}{2}\left(\frac{H^{2}}{Q} \boldsymbol{r}-\frac{H_{h}^{2}}{Q_{h}} \boldsymbol{r}_{h}\right) \cdot \Pi e_{\boldsymbol{p}} d K \\
& =\frac{1}{2} \int_{K}\left(H^{2}\left(\frac{\boldsymbol{r}}{Q}-\frac{\boldsymbol{r}_{h}}{Q_{h}}\right)+\frac{\boldsymbol{r}_{h}}{Q_{h}}\left(H^{2}-H_{h}^{2}\right)\right) \cdot \Pi \boldsymbol{e}_{\boldsymbol{p}} d K .
\end{aligned}
$$

So, we have

$$
|(I I I)| \leq \epsilon \int_{K}\left|\Pi e_{\boldsymbol{p}}\right|^{2} d K+C\left(\int_{K} \boldsymbol{e}_{H}^{2} Q_{h} d K+N_{h}^{K}(t)\right),
$$

where $C$ depends on $\epsilon$ and $\|H\|_{\infty}$. Summing up all the elements $K$, we get

$$
\left.\left|\sum_{K}(I I I)\right| \leq \epsilon\left\|\Pi e_{p}\right\|_{\Omega}^{2}+C\left(N_{h}(t)+\left\|e_{H} \sqrt{Q_{h}}\right\|_{\Omega}^{2}\right)\right) .
$$

- Estimate $(I V)$.

Recalling definition of the projection and fluxes, we obtain $\sum_{K}(I V)$ 


$$
\begin{aligned}
& =\sum_{K}\left(\int_{K}(\boldsymbol{s}-\Pi \boldsymbol{s}) \cdot \Pi \boldsymbol{e}_{\boldsymbol{p}} d K-\int_{K}(\boldsymbol{v}-\Pi \boldsymbol{v}) \cdot \Pi \boldsymbol{e}_{\boldsymbol{p}} d K-\int_{K}(\boldsymbol{p}-\Pi \boldsymbol{p}) \cdot\left(\Pi \boldsymbol{e}_{\boldsymbol{s}}-\Pi \boldsymbol{e}_{\boldsymbol{v}}\right) d K\right) \\
& -\sum_{K} \int_{K}(W-P W) \nabla \cdot\left(\Pi \boldsymbol{e}_{\boldsymbol{s}}-\Pi \boldsymbol{e}_{\boldsymbol{v}}\right) d K+\sum_{K} \int_{\partial K}(\widehat{W-P W})\left(\Pi \boldsymbol{e}_{\boldsymbol{s}}-\Pi \boldsymbol{e}_{\boldsymbol{v}}\right) \cdot \boldsymbol{\nu} d s .
\end{aligned}
$$

In one-dimension, because the choice of numerical flues and the definition of the projection $P^{+-}$we know $P W=P^{-} W, \quad \Pi=P^{+}$and $\widehat{P W}=P W^{-}$, then

$$
\begin{aligned}
& -\sum_{K} \int_{K}(W-P W) \nabla \cdot\left(\Pi \boldsymbol{e}_{\boldsymbol{s}}-\Pi \boldsymbol{e}_{\boldsymbol{v}}\right) d K \\
& =-\sum_{K} \int_{K}\left(W-P^{-} W\right) \nabla \cdot\left(P^{+} \boldsymbol{e}_{\boldsymbol{s}}-P^{+} \boldsymbol{e}_{\boldsymbol{v}}\right) d K \\
& =0,
\end{aligned}
$$

due to the the property of the projection (2.1)and (2.2).

$$
\begin{aligned}
& \sum_{K} \int_{\partial K}(\widehat{W-P} W)\left(\Pi \boldsymbol{e}_{\boldsymbol{s}}-\Pi \boldsymbol{e}_{\boldsymbol{v}}\right) \cdot \boldsymbol{\nu} d s \\
& =\sum_{i}\left(W\left(x_{i+\frac{1}{2}}\right)-P^{-} W\left(x_{i+\frac{1}{2}}^{-}\right)\left(P^{+} \boldsymbol{e}_{\boldsymbol{s}}-P^{+} \boldsymbol{e}_{\boldsymbol{v}}\right)\left(x_{i+\frac{1}{2}}^{-}\right)\right. \\
& -\sum_{i}\left(W\left(x_{i-\frac{1}{2}}\right)-P^{-} W\left(x_{i-\frac{1}{2}}^{-}\right)\right)\left(P^{+} \boldsymbol{e}_{\boldsymbol{s}}-P^{+} \boldsymbol{e}_{\boldsymbol{v}}\right)\left(x_{i-\frac{1}{2}}^{+}\right) \\
& =0
\end{aligned}
$$

where the last step is due to the projection (2.2). So we have

$$
\begin{gathered}
\sum_{K}(I V)=\sum_{K}\left(\int_{K}(\boldsymbol{s}-\Pi \boldsymbol{s}) \cdot \Pi \boldsymbol{e}_{\boldsymbol{p}} d K-\int_{K}(\boldsymbol{v}-\Pi \boldsymbol{v}) \cdot \Pi \boldsymbol{e}_{\boldsymbol{p}} d K\right. \\
\left.-\int_{K}(\boldsymbol{p}-\Pi \boldsymbol{p}) \cdot\left(\Pi \boldsymbol{e}_{\boldsymbol{s}}-\Pi \boldsymbol{e}_{\boldsymbol{v}}\right) d K\right) .
\end{gathered}
$$

In multi-dimension, recalling Lemma 2.1, both cases we have estimates as follows by using Cauchy-Schwarz inequality.

$$
\left|\sum_{K}(I V)\right| \leq \epsilon\left(\left\|\boldsymbol{e}_{\boldsymbol{p}}\right\|_{\Omega}^{2}+\left\|\boldsymbol{e}_{\boldsymbol{s}}\right\|_{\Omega}^{2}+\left\|\boldsymbol{e}_{\boldsymbol{v}}\right\|_{\Omega}^{2}\right)+C h^{2 k+2},
$$

with $C$ depending on $\epsilon$ and $\|u\|_{H^{k+4}(\Omega)}$. And $\epsilon>0$ is any positive constant. Using Lemma 5.4 and Lemma 5.5, we obtain

$$
\left|\sum_{K}(I V)\right| \leq \epsilon\left\|\boldsymbol{e}_{\boldsymbol{p}}\right\|_{\Omega}^{2}+C\left(N_{h}(t)+h^{2 k+2}+\int_{\Omega} \boldsymbol{e}_{H}^{2} Q_{h} d \Omega\right) .
$$

where $C$ depends on $\epsilon,\|u\|_{H^{k+4}(\Omega)},\|\boldsymbol{r}\|_{\infty},\|H\|_{\infty}$.

Collecting $(I),(I I),(I I I),(I V)$, we obtain

$$
\begin{aligned}
& \left|\sum_{K}((I)+(I I)+(I I I)+(I V))\right| \\
& \leq \epsilon\left\|\boldsymbol{e}_{\boldsymbol{p}}\right\|_{\Omega}^{2}+\epsilon \sum_{K} \int_{K} \frac{\boldsymbol{e}_{u_{t}}^{2}}{Q_{h}} d K+C\left(N_{h}(t)+h^{2 k+2}+\int_{\Omega} \boldsymbol{e}_{H}^{2} Q_{h} d \Omega\right) .
\end{aligned}
$$

with $C$ depending on $\epsilon,\|u\|_{H^{k+4}(\Omega)},\|\boldsymbol{r}\|_{\infty},\|H\|_{\infty},\left\|u_{t}\right\|_{\infty}$. 
Next, we try to use the left hand of (A.9) to get control of $\left\|\boldsymbol{e}_{\boldsymbol{p}}\right\|_{\Omega}^{2}$. Adding and subtracting $\boldsymbol{E}\left(\boldsymbol{r}_{h}\right) \boldsymbol{p} \cdot \boldsymbol{e}_{\boldsymbol{p}}$, we have

$$
\begin{aligned}
& \int_{K}\left(\boldsymbol{E}(\boldsymbol{r}) \boldsymbol{p}-\boldsymbol{E}\left(\boldsymbol{r}_{h}\right) \boldsymbol{p}_{h}\right) \cdot \boldsymbol{e}_{\boldsymbol{p}} d K \\
& =\int_{K}\left(\boldsymbol{E}(\boldsymbol{r})-\boldsymbol{E}\left(\boldsymbol{r}_{h}\right)\right) \boldsymbol{p} \cdot \boldsymbol{e}_{\boldsymbol{p}} d K+\int_{K} \boldsymbol{E}\left(\boldsymbol{r}_{h}\right) \boldsymbol{e}_{\boldsymbol{p}} \cdot \boldsymbol{e}_{\boldsymbol{p}} d K .
\end{aligned}
$$

We estimate the first term of the right hand of (A.10). In view of the definition of $\boldsymbol{E}(\boldsymbol{r})$

$$
\left(\boldsymbol{E}(\boldsymbol{r})-\boldsymbol{E}\left(\boldsymbol{r}_{h}\right)\right) \boldsymbol{p} \cdot \boldsymbol{e}_{\boldsymbol{p}}=\boldsymbol{p} \cdot \boldsymbol{e}_{\boldsymbol{p}}\left(\frac{1}{Q}-\frac{1}{Q_{h}}\right)-\left(\boldsymbol{p}^{T}\left(\frac{\boldsymbol{r} \otimes \boldsymbol{r}}{Q^{3}}-\frac{\boldsymbol{r}_{h} \otimes \boldsymbol{r}_{h}}{Q_{h}^{3}}\right)\right) \cdot \boldsymbol{e}_{\boldsymbol{p}} .
$$

Let $z=\frac{r}{Q}, z_{h}=\frac{r_{h}}{Q_{h}}$. By Lemma 4.1, we have

$$
\left|z-z_{h}\right| \leq\left|\gamma-\gamma_{h}\right|
$$

Using the triangle inequality, we get

$$
\begin{aligned}
\frac{\boldsymbol{r} \otimes \boldsymbol{r}}{Q^{3}}-\frac{\boldsymbol{r}_{h} \otimes \boldsymbol{r}_{h}}{Q_{h}^{3}} & =\frac{z \otimes z}{Q}-\frac{z_{h} \otimes z_{h}}{Q_{h}} \\
& =\frac{\left(z-z_{h}\right) \otimes z}{Q}+\left(z_{h} \otimes z\right)\left(\frac{1}{Q}-\frac{1}{Q_{h}}\right)+\frac{z_{h} \otimes\left(z-z_{h}\right)}{Q_{h}} .
\end{aligned}
$$

So we obtain

$$
\left|\frac{\boldsymbol{r} \otimes \boldsymbol{r}}{Q^{3}}-\frac{\boldsymbol{r}_{h} \otimes \boldsymbol{r}_{h}}{Q_{h}^{3}}\right| \leq 3\left|\gamma-\gamma_{h}\right| .
$$

With the help of above estimate, we get

$$
\begin{aligned}
& \left|\int_{K}\left(\boldsymbol{E}(\boldsymbol{r})-\boldsymbol{E}\left(\boldsymbol{r}_{h}\right)\right) \boldsymbol{p} \cdot \boldsymbol{e}_{\boldsymbol{p}} d K\right| \\
& \leq \int_{K} 4|\boldsymbol{p}|\left|\boldsymbol{e}_{\boldsymbol{p}}\right|\left|\boldsymbol{\gamma}-\gamma_{h}\right| d K \\
& =\int_{K} 4|\boldsymbol{p}| \frac{\left|\boldsymbol{e}_{\boldsymbol{p}}\right|}{{\sqrt{Q_{h}}}^{3}}\left|\boldsymbol{\gamma}-\gamma_{h}\right|{\sqrt{Q_{h}}}^{3} d K \\
& \leq \epsilon \int_{K} \frac{\left|\boldsymbol{e}_{\boldsymbol{p}}\right|^{2}}{Q_{h}{ }^{3}} d K+C \int_{K}\left|\boldsymbol{\gamma}-\gamma_{h}\right|^{2} Q_{h}^{3} d K,
\end{aligned}
$$

where $C$ depends on $\epsilon,\|\boldsymbol{p}\|_{\infty}$. Recalling Lemma 4.2 and the definition of $N_{h}^{K}(t)$, we get,

$$
\left|\int_{K}\left(\boldsymbol{E}(\boldsymbol{r})-\boldsymbol{E}\left(\boldsymbol{r}_{h}\right)\right) \boldsymbol{p} \cdot \boldsymbol{e}_{\boldsymbol{p}} d K\right| \leq \epsilon \int_{K} \boldsymbol{E}\left(\boldsymbol{r}_{h}\right) \boldsymbol{e}_{\boldsymbol{p}} \cdot \boldsymbol{e}_{\boldsymbol{p}} d K+C N_{h}^{K}(t)
$$

with $C$ depending on $\epsilon,\|\boldsymbol{p}\|_{\infty}$ and $\|\boldsymbol{r}\|_{\infty}$.

Taking $\epsilon=\frac{1}{2}$, we get

$$
\int_{K}\left(\boldsymbol{E}(\boldsymbol{r})-\boldsymbol{E}\left(\boldsymbol{r}_{h}\right)\right) \boldsymbol{p} \cdot \boldsymbol{e}_{\boldsymbol{p}} d K \geq-\frac{1}{2} \int_{K} \boldsymbol{E}\left(\boldsymbol{r}_{h}\right) \boldsymbol{e}_{\boldsymbol{p}} \cdot \boldsymbol{e}_{\boldsymbol{p}} d K-C N_{h}^{K}(t) .
$$

Finally, we obtain

$$
\int_{K}\left(\boldsymbol{E}(\boldsymbol{r}) \boldsymbol{p}-\boldsymbol{E}\left(\boldsymbol{r}_{h}\right) \boldsymbol{p}_{h}\right) \cdot \boldsymbol{e}_{\boldsymbol{p}} d K \geq \frac{1}{2} \int_{K} \boldsymbol{E}\left(\boldsymbol{r}_{h}\right) \boldsymbol{e}_{\boldsymbol{p}} \cdot \boldsymbol{e}_{\boldsymbol{p}} d K-C N_{h}^{K}(t) .
$$

Summing up all the elements $K$ and using the bound of $(I)-(I V)$ and Lemma 4.2 yields the result. 
A.5. Proof of Lemma 5.9. Using (A.1) and the definition of $Q$ and $Q_{h}$, we have

$$
\begin{aligned}
\left|\gamma-\gamma_{h}\right|^{2} & =\left|\frac{1}{Q}-\frac{1}{Q_{h}}\right|^{2}+\left|\frac{\boldsymbol{r}}{Q}-\frac{\boldsymbol{r}_{h}}{Q_{h}}\right|^{2} \\
& =\frac{1+|\boldsymbol{r}|^{2}}{Q^{2}}+\frac{1+\left|\boldsymbol{r}_{h}\right|^{2}}{Q_{h}^{2}}-\frac{2}{Q Q_{h}}-\frac{2 \boldsymbol{r} \cdot \boldsymbol{r}_{h}}{Q Q_{h}} \\
& =2-2 \frac{1+\boldsymbol{r} \cdot \boldsymbol{r}_{h}}{Q Q_{h}} .
\end{aligned}
$$

Clearly, we get

$$
\frac{1}{2}\left|\gamma-\gamma_{h}\right|^{2}=1-\frac{1+\boldsymbol{r} \cdot \boldsymbol{r}_{h}}{Q Q_{h}} .
$$

Now, we use (A.1) to realize that

$$
\begin{aligned}
\frac{1}{2} \partial_{t}\left(\left|\gamma-\gamma_{h}\right|^{2} Q_{h}\right) & =\partial_{t}\left(\left(1-\frac{1+\boldsymbol{r} \cdot \boldsymbol{r}_{h}}{Q Q_{h}}\right) Q_{h}\right) \\
& =\partial_{t}\left(Q_{h}\right)-\partial_{t}\left(\frac{1}{Q}\right)-\partial_{t}\left(\frac{\boldsymbol{r} \cdot \boldsymbol{r}_{h}}{Q}\right) \\
& =\frac{\boldsymbol{r}_{h} \cdot\left(\boldsymbol{r}_{h}\right)_{t}}{Q_{h}}+\frac{\boldsymbol{r} \cdot \boldsymbol{r}_{t}}{Q^{3}}\left(1+\boldsymbol{r} \cdot \boldsymbol{r}_{h}\right)-\frac{1}{Q}\left(\boldsymbol{r} \cdot\left(\boldsymbol{r}_{h}\right)_{t}+\boldsymbol{r}_{h} \cdot \boldsymbol{r}_{t}\right) \\
& =\left(\frac{\boldsymbol{r}}{Q}-\frac{\boldsymbol{r}_{h}}{Q_{h}}\right) \cdot\left(\boldsymbol{r}_{t}-\left(\boldsymbol{r}_{h}\right)_{t}\right)-\boldsymbol{r}_{t} \cdot\left(\frac{\boldsymbol{r}}{Q}-\frac{\boldsymbol{r}_{h}}{Q_{h}}+\frac{\boldsymbol{r}_{h}}{Q}-\frac{1+\boldsymbol{r} \cdot \boldsymbol{r}_{h}}{Q^{2}} \frac{\boldsymbol{r}}{Q}\right) \\
& =\left(\frac{\boldsymbol{r}}{Q}-\frac{\boldsymbol{r}_{h}}{Q_{h}}\right) \cdot\left(\boldsymbol{r}_{t}-\left(\boldsymbol{r}_{h}\right)_{t}\right)-(I I I) .
\end{aligned}
$$

Here,

$$
\begin{aligned}
(I I I) & =\boldsymbol{r}_{t} \cdot\left(\frac{\boldsymbol{r}}{Q}-\frac{\boldsymbol{r}_{h}}{Q_{h}}+\frac{\boldsymbol{r}_{h}}{Q}-\frac{1+\boldsymbol{r} \cdot \boldsymbol{r}_{h}}{Q^{2}} \frac{\boldsymbol{r}}{Q}\right) \\
& =\boldsymbol{r}_{t} \cdot\left(\frac{\boldsymbol{r}}{Q}-\frac{\boldsymbol{r}_{h}}{Q_{h}}\right)\left(\frac{1}{Q_{h}}-\frac{1}{Q}\right) Q_{h}+\boldsymbol{r}_{t} \cdot \frac{\boldsymbol{r}}{Q^{2}}\left(1-\frac{1+\boldsymbol{r} \cdot \boldsymbol{r}_{h}}{Q Q_{h}}\right) Q_{h} .
\end{aligned}
$$

By Lemma 4.1 and equation (A.11), we get

$$
\int_{K}(I I I) d K \geq-\left\|\boldsymbol{r}_{t}\right\|_{\infty} N_{h}^{K}(t)
$$

We proceed as follows with the help of the above equality.

$$
\begin{aligned}
& \int_{K}\left(\frac{\boldsymbol{r}}{Q} \cdot \boldsymbol{e}_{\boldsymbol{r}_{t}}-\frac{\boldsymbol{r}_{h}}{Q_{h}} \cdot \boldsymbol{e}_{\boldsymbol{r}_{t}}\right) d K \\
& =\int_{K}\left(\frac{\boldsymbol{r}}{Q}-\frac{\boldsymbol{r}_{h}}{Q_{h}}\right) \cdot \boldsymbol{e}_{\boldsymbol{r}_{t}} d K \\
& =\int_{K}\left(\frac{\boldsymbol{r}}{Q}-\frac{\boldsymbol{r}_{h}}{Q_{h}}\right) \cdot\left(\boldsymbol{r}_{t}-\left(\boldsymbol{r}_{h}\right)_{t}\right) d K \\
& =\frac{1}{2} \partial_{t} \int_{K}\left(\left|\boldsymbol{\gamma}-\gamma_{h}\right|^{2} Q_{h}\right) d K+\int_{K}(I I I) d K \\
& \geq \frac{1}{2} \frac{d}{d t} \int_{K}\left(\left|\boldsymbol{\gamma}-\gamma_{h}\right|^{2} Q_{h}\right) d K-\left\|\boldsymbol{r}_{t}\right\|_{\infty} N_{h}^{K}(t) .
\end{aligned}
$$

(5.14) follows by taking $C=\left\|\boldsymbol{r}_{t}\right\|_{\infty}$. 
A.6. Proof of Lemma 5.10. Differentiating (3.4h) with respect to time and combining with (3.4f)-(3.4g), we have

$$
\begin{aligned}
& \int_{K} H_{h} \vartheta d K+\int_{K} \boldsymbol{q}_{h} \cdot \nabla \vartheta d K-\int_{\partial K} \widehat{\boldsymbol{q}_{h}} \cdot \boldsymbol{\nu} \vartheta d s=0, \\
& \int_{K} \boldsymbol{q}_{h} \cdot \boldsymbol{\rho} d K-\int_{K} \frac{\boldsymbol{r}_{h}}{Q_{h}} \cdot \boldsymbol{\rho} d K=0, \\
& \int_{K}\left(\boldsymbol{r}_{h}\right)_{t} \cdot \boldsymbol{\zeta} d K+\int_{K}\left(u_{h}\right)_{t} \nabla \cdot \boldsymbol{\zeta} d K-\int_{\partial K} \widehat{\left(u_{h}\right)_{t}} \boldsymbol{\nu} \cdot \boldsymbol{\zeta} d s=0 .
\end{aligned}
$$

Choosing $\vartheta=P e_{u_{t}}, \rho=-\Pi e_{r_{t}}, \zeta=\Pi e_{q}$, we obtain the error equations

$$
\begin{aligned}
& \int_{K} P \boldsymbol{e}_{H} P \boldsymbol{e}_{u_{t}} d K+\int_{K} \Pi \boldsymbol{e}_{\boldsymbol{q}} \cdot \nabla P \boldsymbol{e}_{u_{t}} d K-\int_{\partial K} P \boldsymbol{e}_{u_{t}} \widehat{\Pi \boldsymbol{e}_{\boldsymbol{q}}} \cdot \boldsymbol{\nu} d s \\
& +\int_{K}\left(H-H_{h}\right) P \boldsymbol{e}_{u_{t}} d K+\int_{K}(\boldsymbol{q}-\Pi \boldsymbol{q}) \cdot \nabla P \boldsymbol{e}_{u_{t}} d K-\int_{\partial K} P \boldsymbol{e}_{u_{t}}(\widehat{\boldsymbol{q - \Pi}}) \cdot \boldsymbol{\nu} d s=0 \\
& -\int_{K} \Pi \boldsymbol{e}_{\boldsymbol{q}} \cdot \boldsymbol{e}_{\boldsymbol{r}_{t}} d K+\int_{K}\left(\frac{\boldsymbol{r}}{Q}-\frac{\boldsymbol{r}_{h}}{Q_{h}}\right) \cdot \Pi \boldsymbol{e}_{\boldsymbol{r}_{t}} d K=0 \\
& \int_{K} \Pi \boldsymbol{e}_{\boldsymbol{r}_{t}} \cdot \Pi \boldsymbol{e}_{\boldsymbol{q}} d K+\int_{K} P \boldsymbol{e}_{u_{t}} \nabla \cdot \Pi \boldsymbol{e}_{\boldsymbol{q}} d K-\int_{\partial K} \widehat{P \boldsymbol{e}_{u_{t}}} \Pi \boldsymbol{e}_{\boldsymbol{q}} \cdot \boldsymbol{\nu} d s \\
& +\int_{K}\left(\boldsymbol{r}_{t}-\Pi \boldsymbol{r}_{t}\right) \cdot \Pi e_{\boldsymbol{q}} d K+\int_{K}\left(u_{t}-P u_{t}\right) \nabla \cdot \Pi \boldsymbol{e}_{\boldsymbol{q}} d K-\int_{\partial K}\left(\widehat{u_{t}-P u_{t}}\right) \Pi \boldsymbol{e}_{\boldsymbol{q}} \cdot \boldsymbol{\nu} d s=0 .
\end{aligned}
$$

In view of,

$$
e_{r}=r-\Pi r+\Pi e_{r},
$$

we derive

$$
\begin{aligned}
& \int_{K}\left(\frac{\boldsymbol{r}}{Q}-\frac{\boldsymbol{r}_{h}}{Q_{h}}\right) \cdot \boldsymbol{e}_{\boldsymbol{r}_{t}} d K \\
& =\int_{K}\left(\frac{\boldsymbol{r}}{Q}-\frac{\boldsymbol{r}_{h}}{Q_{h}}\right) \cdot\left(\boldsymbol{r}_{t}-\Pi \boldsymbol{r}_{t}\right) d K \\
& -\int_{K} P \boldsymbol{e}_{H} P \boldsymbol{e}_{u_{t}} d K-\int_{K}(H-P H) P \boldsymbol{e}_{u_{t}} d K-\int_{K}\left(\boldsymbol{r}_{t}-\Pi \boldsymbol{r}_{t}\right) \cdot \Pi \boldsymbol{e}_{\boldsymbol{q}} d K \\
& -\int_{K}(\boldsymbol{q}-\Pi \boldsymbol{q}) \cdot \nabla P \boldsymbol{e}_{u_{t}} d K+\int_{\partial K} P \boldsymbol{e}_{u_{t}}(\widehat{\boldsymbol{q}-\Pi \boldsymbol{q}}) \cdot \boldsymbol{\nu} d s \\
& -\int_{K}\left(u_{t}-P u_{t}\right) \nabla \cdot \Pi \boldsymbol{e}_{\boldsymbol{q}} d K+\int_{\partial K}\left(\widehat{u_{t}-P u_{t}}\right) \Pi \boldsymbol{e}_{\boldsymbol{q}} \cdot \boldsymbol{\nu} d s
\end{aligned}
$$

We firstly sum up all the elements $K$. Then we obtain the result by recalling the projection we choose and using Lemma 2.1, Lemma 5.9.

A.7. Proof of Lemma 5.12. Consider the nonlinear term

$$
\begin{aligned}
& \left(\boldsymbol{E}(\boldsymbol{r}) \boldsymbol{r}_{t}-\boldsymbol{E}\left(\boldsymbol{r}_{h}\right)\left(\boldsymbol{r}_{h}\right)_{t}\right) \cdot \Pi e_{\boldsymbol{p}}-\left(\boldsymbol{E}(\boldsymbol{r}) \boldsymbol{p}-\boldsymbol{E}\left(\boldsymbol{r}_{h}\right) \boldsymbol{p}_{h}\right) \cdot \Pi e_{\boldsymbol{r}_{t}} \\
& =\left(\boldsymbol{E}(\boldsymbol{r}) \boldsymbol{r}_{t}-\boldsymbol{E}(\Pi \boldsymbol{r}) \Pi \boldsymbol{r}_{t}\right) \cdot \Pi e_{\boldsymbol{p}}-(\boldsymbol{E}(\boldsymbol{r}) \boldsymbol{p}-\boldsymbol{E}(\Pi \boldsymbol{r}) \Pi \boldsymbol{p}) \cdot \Pi e_{\boldsymbol{r}_{t}} \\
& +\left(\left(\boldsymbol{E}(\Pi \boldsymbol{r}) \Pi \boldsymbol{r}_{t}-\boldsymbol{E}\left(\boldsymbol{r}_{h}\right)\left(\boldsymbol{r}_{h}\right)_{t}\right) \cdot \Pi \boldsymbol{e}_{\boldsymbol{p}}-\left(\boldsymbol{E}(\Pi \boldsymbol{r}) \Pi \boldsymbol{p}-\boldsymbol{E}\left(\boldsymbol{r}_{h}\right) \boldsymbol{p}_{h}\right) \cdot \Pi \boldsymbol{e}_{\boldsymbol{r}_{t}}\right) \\
& =(V)+(V I)+(V I I) .
\end{aligned}
$$

In view of the Lemma 4.2 , obviously we have

$$
\left|\sum_{K} \int_{K}(V) d K\right| \leq \epsilon\left\|\Pi e_{p}\right\|_{\Omega}^{2}+C h^{2 k+2},
$$


where $C$ depends on $\epsilon$ and $\left\|\boldsymbol{r}_{t}\right\|_{H^{k+1}(\Omega)}$.

$$
\begin{aligned}
(V I) & =-(\boldsymbol{E}(\boldsymbol{r}) \boldsymbol{p}-\boldsymbol{E}(\Pi \boldsymbol{r}) \Pi \boldsymbol{p}) \cdot \Pi e_{\boldsymbol{r}_{t}} \\
& =-\frac{\partial}{\partial t}\left((\boldsymbol{E}(\boldsymbol{r}) \boldsymbol{p}-\boldsymbol{E}(\Pi \boldsymbol{r}) \Pi \boldsymbol{p}) \cdot \Pi e_{\boldsymbol{r}}\right)+\frac{\partial}{\partial t}(\boldsymbol{E}(\boldsymbol{r}) \boldsymbol{p}-\boldsymbol{E}(\Pi \boldsymbol{r}) \Pi \boldsymbol{p}) \cdot \Pi e_{\boldsymbol{p}} \\
& \geq-\frac{\partial}{\partial t}\left((\boldsymbol{E}(\boldsymbol{r}) \boldsymbol{p}-\boldsymbol{E}(\Pi \boldsymbol{r}) \Pi \boldsymbol{p}) \cdot \Pi e_{\boldsymbol{r}}\right)-\epsilon\left\|\Pi \boldsymbol{e}_{\boldsymbol{p}}\right\|_{\Omega}^{2}-C h^{2 k+2},
\end{aligned}
$$

where $C$ depends on $\epsilon,\|\boldsymbol{p}\|_{H^{k+1}},\|\boldsymbol{r}\|_{H^{k+1}}$.

Next, we estimate $(V I I)$. This term is the same as the paper of the Decklnick [12]. So we just cite their result

$$
\begin{aligned}
& \sum_{K} \int_{K}(V I I) d K \\
& \geq-\frac{d}{d t} \int_{\Omega}\left(\left(\frac{Q_{h}}{\widetilde{Q_{h}}}-1\right)\left(\widetilde{\gamma_{h}}-\gamma_{h}\right)-\frac{1}{2}\left(\frac{Q_{h}}{\widetilde{Q_{h}}}\left|\widetilde{\gamma_{h}}-\gamma_{h}\right|^{2} \widetilde{\gamma_{h}}\right)\right) \cdot(\Pi p, 0)^{T} d \Omega \\
& -C\left\|\Pi e_{\boldsymbol{r}}\right\|_{\Omega}^{2}-\epsilon\left\|\Pi e_{\boldsymbol{p}}\right\|_{\Omega}^{2},
\end{aligned}
$$

where $C$ depends on $\epsilon,\|\boldsymbol{r}\|_{\infty},\|\boldsymbol{p}\|_{\infty}$. And denote

$$
\widetilde{Q_{h}}=\sqrt{1+|\Pi \boldsymbol{r}|^{2}}, \quad \widetilde{\gamma_{h}}=\frac{(-\Pi \boldsymbol{r}, 1)^{T}}{\widetilde{Q_{h}}} .
$$

Combining $(V),(V I),(V I I)$ gives the results.

\section{A.8. Proof of Lemma 5.13. We consider}

$$
\mathcal{Z}_{1}+\mathcal{Z}_{2}=\left(Q H-Q_{h} H_{h}\right) P \boldsymbol{e}_{H_{t}}+\frac{1}{2}\left(\frac{H^{2}}{Q} \boldsymbol{r}-\frac{H_{h}^{2}}{Q_{h}} \boldsymbol{r}_{h}\right) \cdot \Pi \boldsymbol{e}_{\boldsymbol{r}_{t}} .
$$

In view of $P \boldsymbol{e}_{H_{t}}=\left(H_{t}-\left(H_{h}\right)_{t}\right)-\left(H_{t}-P H_{t}\right)$, We rewrite $\mathcal{Z}_{1}$ as follows

$$
\begin{aligned}
\mathcal{Z}_{1} & =\left(Q H-Q_{h} H_{h}\right) P e_{H_{t}} \\
& =\left(\left(H-H_{h}\right) Q_{h}+\left(Q-Q_{h}\right) H\right)\left(\left(H_{t}-\left(H_{h}\right)_{t}\right)-\left(H_{t}-P H_{t}\right)\right) \\
& =\left(H-H_{h}\right)\left(H_{t}-\left(H_{h}\right)_{t}\right) Q_{h}-\left(H-H_{h}\right)\left(H_{t}-P H_{t}\right) Q_{h} \\
& -\left(Q-Q_{h}\right)\left(H_{t}-P H_{t}\right) H+\left(Q-Q_{h}\right)\left(H_{t}-\left(H_{h}\right)_{t}\right) H \\
& =\frac{1}{2} \frac{\partial}{\partial t}\left(\left(H-H_{h}\right)^{2} Q_{h}\right)-\frac{1}{2}\left(H-H_{h}\right)^{2} Q_{h t} \\
& -\left(H-H_{h}\right)\left(H_{t}-P H_{t}\right) Q_{h}-\left(Q-Q_{h}\right)\left(H_{t}-P H_{t}\right) H \\
& +\frac{\partial}{\partial t}\left(\left(Q-Q_{h}\right)\left(H-H_{h}\right) H\right)-\left(Q-Q_{h}\right)\left(H-H_{h}\right) H_{t}-\left(Q_{t}-Q_{h t}\right)\left(H-H_{h}\right) H .
\end{aligned}
$$

So we have

$$
\begin{aligned}
\mathcal{Z}_{1}+\mathcal{Z}_{2}= & \frac{1}{2} \frac{\partial}{\partial t}\left(\left(H-H_{h}\right)^{2} Q_{h}\right)-\left(H-H_{h}\right)\left(H_{t}-P H_{t}\right) Q_{h}-\left(Q-Q_{h}\right)\left(H_{t}-P H_{t}\right) H \\
& +\frac{\partial}{\partial t}\left(\left(Q-Q_{h}\right)\left(H-H_{h}\right) H\right)-\left(Q-Q_{h}\right)\left(H-H_{h}\right) H_{t}+\mathcal{Z}_{3},
\end{aligned}
$$

where

$$
\mathcal{Z}_{3}=\frac{1}{2}\left(\frac{H^{2}}{Q} \boldsymbol{r}-\frac{H_{h}^{2}}{Q_{h}} \boldsymbol{r}_{h}\right) \cdot \Pi \boldsymbol{e}_{\boldsymbol{r}_{t}}-\frac{1}{2}\left(H-H_{h}\right)^{2} Q_{h t}-\left(Q_{t}-Q_{h t}\right)\left(H-H_{h}\right) H .
$$

Now, we deal with $\mathcal{Z}_{3}$.

$$
\begin{aligned}
\mathcal{Z}_{3}= & \frac{1}{2}\left(\frac{H^{2}}{Q} \boldsymbol{r}-\frac{H_{h}^{2}}{Q_{h}} \boldsymbol{r}_{h}\right) \cdot\left(\Pi \boldsymbol{r}_{t}-\left(\boldsymbol{r}_{h}\right)_{t}\right)-\frac{1}{2}\left(H^{2} Q_{h t}-2 H H_{h} Q_{h t}+H_{h}^{2} Q_{h t}\right) \\
& -\left(H^{2} Q_{t}-H^{2} Q_{h t}-H H_{h} Q_{t}+H H_{h} Q_{h t}\right)
\end{aligned}
$$




$$
\begin{aligned}
& =\frac{1}{2} \frac{H^{2}}{Q} \boldsymbol{r} \cdot \Pi \boldsymbol{r}_{t}-\frac{1}{2} \frac{H^{2}}{Q} \boldsymbol{r} \cdot\left(\boldsymbol{r}_{h}\right)_{t}-\frac{1}{2} \frac{H_{h}^{2}}{Q_{h}} \boldsymbol{r}_{h} \cdot \Pi \boldsymbol{r}_{t}+\frac{1}{2} H^{2} Q_{h t}-H^{2} Q_{t}+H H_{h} Q_{t} \\
& =\mathcal{S}_{1}+\mathcal{S}_{2}+\mathcal{S}_{3}+\mathcal{S}_{4}+\mathcal{S}_{5}+\mathcal{S}_{6} .
\end{aligned}
$$

We observe that

$$
\begin{aligned}
\mathcal{S}_{2} & =-\frac{1}{2} \frac{H^{2}}{Q} \boldsymbol{r} \cdot\left(\boldsymbol{r}_{h}\right)_{t} \\
& =-\frac{1}{2} H^{2} \partial_{t}\left(\frac{\boldsymbol{r} \cdot \boldsymbol{r}_{h}}{Q}\right)+\frac{1}{2} H^{2} \frac{\boldsymbol{r}_{t} \cdot \boldsymbol{r}_{h}}{Q}-\frac{1}{2} H^{2} Q_{t} \frac{\boldsymbol{r} \cdot \boldsymbol{r}_{h}}{Q^{2}} .
\end{aligned}
$$

A simple calculation yields

$$
\frac{1}{2}\left|\gamma-\gamma_{h}\right|^{2}=1-\frac{1+\boldsymbol{r} \cdot \boldsymbol{r}_{h}}{Q Q_{h}} .
$$

Consequently, we have

$$
\boldsymbol{r} \cdot \boldsymbol{r}_{h}=\left(1-\frac{1}{2}\left|\gamma-\gamma_{h}\right|^{2}\right) Q Q_{h}-1 .
$$

So, we get

$$
\begin{aligned}
\mathcal{S}_{2}= & -\frac{1}{2} H^{2} \partial_{t}\left(\frac{\boldsymbol{r} \cdot \boldsymbol{r}_{h}}{Q}\right)+\frac{1}{2} H^{2} \frac{\boldsymbol{r}_{t} \cdot \boldsymbol{r}_{h}}{Q} \\
& -\frac{1}{2} \frac{Q_{h} Q_{t}}{Q} H^{2}+\frac{1}{4} \frac{H^{2} Q_{t}}{Q}\left|\boldsymbol{\gamma}-\boldsymbol{\gamma}_{h}\right|^{2} Q_{h}-\frac{1}{2} H^{2} \partial_{t}\left(\frac{1}{Q}\right) .
\end{aligned}
$$

Note that

$$
\begin{aligned}
\mathcal{S}_{3} & =-\frac{1}{2} \frac{H_{h}^{2}}{Q_{h}} \boldsymbol{r}_{h} \cdot \Pi \boldsymbol{r}_{t} \\
& =-\frac{1}{2}\left(\left(H-H_{h}\right)^{2}+2 H H_{h}-H^{2}\right) \frac{\boldsymbol{r}_{h} \cdot \Pi \boldsymbol{r}_{t}}{Q_{h}} \\
& =-\frac{1}{2} \frac{\boldsymbol{r}_{h} \cdot \Pi \boldsymbol{r}_{t}}{Q_{h}} \boldsymbol{e}_{H}^{2}-H H_{h} \frac{\boldsymbol{r}_{h} \cdot \Pi \boldsymbol{r}_{t}}{Q_{h}}+\frac{1}{2} H^{2} \frac{\boldsymbol{r}_{h} \cdot \Pi \boldsymbol{r}_{t}}{Q_{h}},
\end{aligned}
$$

and

$$
\mathcal{S}_{4}+\mathcal{S}_{5}=\frac{1}{2} H^{2} \partial_{t}\left(Q_{h}-2 Q\right) .
$$

Collecting $\mathcal{S}_{1}, \mathcal{S}_{2}, \mathcal{S}_{3}, \mathcal{S}_{4}, \mathcal{S}_{5}, \mathcal{S}_{6}$ we obtain

$$
\begin{aligned}
& \mathcal{S}_{1}+\mathcal{S}_{2}+\mathcal{S}_{3}+\mathcal{S}_{4}+\mathcal{S}_{5}+\mathcal{S}_{6} \\
& =\frac{H^{2} Q_{t}}{4 Q}\left|\boldsymbol{\gamma}-\gamma_{h}\right|^{2} Q_{h}-\frac{1}{2} \frac{\boldsymbol{r}_{h} \cdot \Pi \boldsymbol{r}_{t}}{Q_{h}} e_{H}^{2}+\frac{1}{2} H^{2} \partial_{t}\left(Q_{h}-2 Q-\frac{\boldsymbol{r} \cdot \boldsymbol{r}_{h}}{Q}-\frac{1}{Q}\right) \\
& +\frac{1}{2} H^{2} \frac{\boldsymbol{r} \cdot \Pi \boldsymbol{r}_{t}}{Q}+\frac{1}{2} H^{2} \frac{\boldsymbol{r}_{t} \cdot \boldsymbol{r}_{h}}{Q}-\frac{1}{2} H^{2} \frac{Q_{h} Q_{t}}{Q}-H H_{h} \frac{\boldsymbol{r}_{h} \cdot \Pi \boldsymbol{r}_{t}}{Q_{h}}+\frac{1}{2} \frac{H^{2}}{Q_{h}} \boldsymbol{r}_{h} \cdot \Pi \boldsymbol{r}_{t}+H H_{h} Q_{t} .
\end{aligned}
$$

Using the relation $\frac{1}{2}\left|\gamma-\gamma_{h}\right|^{2}=1-\frac{1+\boldsymbol{r} \cdot \boldsymbol{r}_{h}}{Q Q_{h}}$, again, we have

$$
\frac{1}{2} H^{2} \partial_{t}\left(Q_{h}-2 Q-\frac{\boldsymbol{r} \cdot \boldsymbol{r}_{h}}{Q}-\frac{1}{Q}\right)=\frac{1}{2} H^{2} \partial_{t}\left(\frac{1}{2}\left|\gamma-\gamma_{h}\right|^{2} Q_{h}\right)-H^{2} Q_{t} .
$$

With the aid of the above equality, we have

$$
\begin{aligned}
& \mathcal{S}_{1}+\mathcal{S}_{2}+\mathcal{S}_{3}+\mathcal{S}_{4}+\mathcal{S}_{5}+\mathcal{S}_{6} \\
& =\frac{H^{2} Q_{t}}{4 Q}\left|\gamma-\gamma_{h}\right|^{2} Q_{h}-\frac{1}{2} \frac{\boldsymbol{r}_{h} \cdot \Pi \boldsymbol{r}_{t}}{Q_{h}} e_{H}^{2}+\frac{1}{2} H^{2} \partial_{t}\left(\frac{1}{2}\left|\gamma-\gamma_{h}\right|^{2} Q_{h}\right)+\mathcal{Z}_{4},
\end{aligned}
$$


where

$$
\begin{aligned}
\mathcal{Z}_{4}= & -H^{2} Q_{t}+\frac{1}{2} H^{2} \frac{\boldsymbol{r} \cdot \Pi \boldsymbol{r}_{t}}{Q}+\frac{1}{2} H^{2} \frac{\boldsymbol{r}_{t} \cdot \boldsymbol{r}_{h}}{Q}-\frac{1}{2} H^{2} \frac{Q_{h} Q_{t}}{Q} \\
& -H H_{h} \frac{\boldsymbol{r}_{h} \cdot \Pi \boldsymbol{r}_{t}}{Q_{h}}+\frac{1}{2} \frac{H^{2}}{Q_{h}} \boldsymbol{r}_{h} \cdot \Pi \boldsymbol{r}_{t}+H H_{h} Q_{t} \\
= & H\left(H_{h}-H\right)\left(Q_{t}-\frac{\boldsymbol{r}_{h} \cdot \Pi \boldsymbol{r}_{t}}{Q_{h}}\right)+\frac{1}{2} H^{2}\left(\frac{\boldsymbol{r}}{Q}-\frac{\boldsymbol{r}_{h}}{Q_{h}}\right) \cdot\left(\Pi \boldsymbol{r}_{t}-\boldsymbol{r}_{t}\right) \\
& +\frac{1}{2} H^{2}\left(\boldsymbol{r}_{h}-\boldsymbol{r}\right) \cdot \boldsymbol{r}_{t}\left(\frac{1}{Q}-\frac{1}{Q_{h}}\right)-\frac{1}{2} H^{2} \frac{Q_{t}\left(Q-Q_{h}\right)^{2}}{Q Q_{h}} .
\end{aligned}
$$

We finally obtain

$$
\begin{aligned}
& \int_{K}\left(Q H-Q_{h} H_{h}\right) P \boldsymbol{e}_{H_{t}} d K+\int_{K} \frac{1}{2}\left(\frac{H^{2}}{Q} \boldsymbol{r}-\frac{H_{h}^{2}}{Q_{h}} \boldsymbol{r}_{h}\right) \cdot \Pi \boldsymbol{e}_{\boldsymbol{r}_{t}} d K \\
& =\frac{1}{2} \frac{d}{d t} \int_{K} \boldsymbol{e}_{H}^{2} Q_{h} d K+\frac{d}{d t} \int_{K}\left(Q-Q_{h}\right)\left(H-H_{h}\right) H d K+\int_{K} \frac{1}{2} H^{2} \partial_{t}\left(\frac{1}{2}\left|\boldsymbol{\gamma}-\gamma_{h}\right|^{2} Q_{h}\right) d K \\
& \quad-\int_{K}\left(H-H_{h}\right)\left(H_{t}-P H_{t}\right) Q_{h} d K-\int_{K}\left(Q-Q_{h}\right)\left(H_{t}-P H_{t}\right) H d K \\
& \quad-\int_{K}\left(H-H_{h}\right)\left(Q-Q_{h}\right) H_{t} d K+\int_{K} \frac{H^{2} Q_{t}}{4 Q}\left|\boldsymbol{\gamma}-\boldsymbol{\gamma}_{h}\right|^{2} Q_{h} d K-\int_{K} \frac{\boldsymbol{r}_{h} \cdot \Pi \boldsymbol{r}_{t}}{2 Q_{h}} \boldsymbol{e}_{H}^{2} d K \\
& \quad+\int_{K} H\left(H_{h}-H\right)\left(Q_{t}-\frac{\boldsymbol{r}_{h} \cdot \Pi \boldsymbol{r}_{t}}{Q_{h}}\right) d K+\int_{K} \frac{1}{2} H^{2}\left(\frac{\boldsymbol{r}}{Q}-\frac{\boldsymbol{r}_{h}}{Q_{h}}\right) \cdot\left(\Pi \boldsymbol{r}_{t}-\boldsymbol{r}_{t}\right) d K \\
& \quad+\int_{K} \frac{1}{2} H^{2}\left(\boldsymbol{r}_{h}-\boldsymbol{r}\right) \cdot \boldsymbol{r}_{t}\left(\frac{1}{Q}-\frac{1}{Q_{h}}\right) d K-\int_{K} \frac{H^{2} Q_{t}\left(Q-Q_{h}\right)^{2}}{2 Q Q_{h}} d K .
\end{aligned}
$$

A simple calculation yields the result.

\section{References}

[1] F. Bassi and S. Rebay, A high-order accurate discontinuous finite element method for the numerical solution of the compressible Navier-Stokes equations, J. Comput. Phys., 131 (1997), pp.267-279.

[2] P. Ciarlet, The finite element method for elliptic problem, North Holland, 1975.

[3] B. Cockburn, S. Hou and C.-W. Shu, The Runge-Kutta local projection discontinuous Galerkin finite element method for conservation laws IV: the multidimensional case, Math. Comp., 54 (1990), pp.545-581.

[4] B. Cockburn, S.-Y. Lin and C.-W. Shu, TVB Runge-Kutta local projection discontinuous Galerkin finite element method for conservation laws III: one dimensional systems, J. Comput. Phys., 84 (1989), pp.90-113.

[5] B. Cockburn and C.-W. Shu, TVB Runge-Kutta local projection discontinuous Galerkin finite element method for conservation laws II: general framework, Math. Comp., 52 (1989), pp.411-435.

[6] B. Cockburn and C.-W. Shu, The Runge-Kutta discontinuous Galerkin method for conservation laws V: multidimensional systems, J. Comput. Phys., 141 (1998), pp.199-224.

[7] B. Cockburn and C.-W. Shu, The local discontinuous Galerkin method for time-dependent convection-diffusion systems, SIAM J. Numer. Anal., 35 (1998), pp.2440-2463.

[8] B. Cockburn, G. Kanschat, I. Perugia and D. schötzau, Superconvergence of the local discontinuous Galerkin method for elliptic problems on cartesian grids, SIAM J. Numer. Anal., 39 (2001), pp.264-285.

[9] B. Cockburn and C.-W. Shu, Foreword for the special issue on discontinuous Galerkin method, J. Sci. Comput., 22-23 (2005), pp.1-3.

[10] B. Cockburn and C.-W. Shu, Foreword, J. Sci. Comput., 40 (2009), pp.1-3.

[11] C. Dawson, Foreword for the special issue on discontinuous Galerkin method, Comput. Meth. Appl. Mech. Engrg., 195 (2006), p.3183.

[12] K. Deckelnick and G. Dziuk, Error analysis of a finite element method for the Willmore flow of graphs, Interfaces Free Bound., 8 (2006), pp.21-46. 
[13] B. Dong and C.-W. Shu. Analysis of a local discontinuous Galerkin method for fourth-order time-dependent problems. SIAM J. Numer. Anal., 47 (2009), pp.3240-3268.

[14] J. Hesthaven and T. Warburton. Nodal discontinuous Galerkin methods. Algorithms, analysis, and applications. Springer, 2008.

[15] B. Li. Discontinuous finite elements in fluid dynamics and heat transfer. Springer-Verlag London, 2006.

[16] H. Liu and J. Yan, A local discontinuous Galerkin method for the Korteweg-de Vries equation with boundary effect, J. Comput. Phys., 215 (2006), pp.197-218.

[17] W.H. Reed and T.R. Hill, Triangular mesh method for the neutron transport equation, Technical report LA-UR-73-479, Los Alamos Scientific Laboratory, Los Alamos, NM, 1973.

[18] B. Rivière. Discontinuous Galerkin methods for solving elliptic and parabolic equations. Theory and implementation. SIAM, 2008.

[19] C.-W. Shu. Discontinuous Galerkin methods: general approach and stability, Numerical Solutions of Partial Differential Equations, S. Bertoluzza, S. Falletta, G. Russo and C.-W. Shu, Advanced Courses in Mathematics CRM Barcelona, Pages 149-201. Birkhäuser, Basel, 2009.

[20] Y. Xia, Y. Xu and C.-W. Shu, Local discontinuous Galerkin methods for the Cahn-Hilliard type equations, J. Comput. Phys., 227 (2007), pp. 472-491.

[21] Y. Xia, Y. Xu and C.-W. Shu, Application of the local discontinuous Galerkin method for the Allen-Cahn/Cahn-Hilliard system, Commun. Comput. Phys., 5 (2009), pp.821-835.

[22] Y. Xu and C.-W. Shu, Local discontinuous Galerkin methods for two classes of two dimensional nonlinear wave equations, Physica D, 208 (2005), pp.21-58.

[23] Y. Xu and C.-W. Shu, Local discontinuous Galerkin method for surface diffusion and Willmore flow of graphs, Journal of Scientific Computing, 40 (2009), pp.375-390.

[24] Y. Xu and C.-W. Shu, Local discontinuous Galerkin methods for high-order time-dependent partial differential equations, Communications in Computational Physics, 7 (2010), pp. 1-46.

[25] J. Yan and C.-W. Shu, A local discontinuous Galerkin method for KdV type equations, SIAM J. Numer. Anal., 40 (2002), pp.769-791.

Department of Mathematics, University of Science and Technology of China, Hefei, Anhui 230026, P.R. China.

E-mail: jlyue@mail.ustc.edu.cn

Department of Mathematics, University of Science and Technology of China, Hefei, Anhui 230026, P.R. China.

E-mail: yxu@ustc.edu.cn

$U R L:$ http://staff.ustc.edu.cn/ $\sim \mathrm{yxu}$ 